\title{
On the influence of outer large-scale structures on near-wall turbulence in channel flow
}

\author{
L. Agostini and M. A. Leschziner \\ Department of Aeronautics \\ Imperial College London \\ South Kensington, London, SW7 2AZ, UK
}

(Dated: July 22, 2014)

\begin{abstract}
DNS data for channel flow at $R e_{\tau}=1025$ are used to analyse the interaction between large outer scales in the log-law region - referred to as super-streaks - and the small-scale, streaky, streamwisevelocity fluctuations in the viscosity-affected near-wall layer. The study is inspired by extensive experimental investigations by Mathis, Marusic and Hutchins, culminating in a predictive model that describes, in a supposedly universal manner, the "footprinting" and "modulating" effects of the outer structures on the small-scale near-wall motions. The approach used herein is based on the examination of joint PDFs for the small-scale fluctuations, conditioned on regions of large-scale footprints. The large and small scales are separated by means of the Huang-Hilbert empirical-mode decomposition, the validity of which is demonstrated by way of pre-multiplied energy spectra, correlation maps and energy profiles for both scales. Observations derived from the PDFs then form the basis of assessing the validity of the assumptions underlying the model. Although the present observations support some elements of the model, the results imply that modulation by negative and positive large-scale fluctuations differ greatly - an asymmetric response that is not compatible with the model. The study is thus extended to examining the validity of an alternative proposal, which is based on the assumption that a universal description of the small-scale response to the large-scale motions has to rely on the velocity fluctuations being scaled with the largescales-modified local friction velocity, rather than with the mean value. This proposal is partially supported by the present analysis. Finally, an alternative, new phenomenological model is proposed and examined.
\end{abstract}

Keywords: Channel flow, Direct Numerical Simulation, Turbulent boundary layer 


\section{INTRODUCTION}

There is a substantial body of literature, going back to the 1970's and 80's ${ }^{1,2}$, that deals with the properties of large-scale structures in the outer portions of near-wall shear layers, in general, and with the link between these structures and small-scale 'streaky' patterns in the near-wall region, in particular. More recently, interest in the fundamental mechanisms governing near-wall turbulence has been boosted by the discovery of an outer 'secondary peak' (or plateau) in turbulence energy and its pre-multiplied longitudinal energy spectrum at high Reynolds numbers, and an increased recognition that the small-scale near-wall streaks that governs the skin friction are significantly affected by the large-scale structures that populate the outer layer in the which the secondary energy maximum is observed. The ability to gain insight into these interactions has been substantially aided, in recent years, by influential improvements in experimental, data-processing and scale-resolving-simulation techniques, and the resulting increase in activity, mainly since 2003/4, has spawned numerous papers that deal with various structural aspects of, and related interactions in, near-wall turbulence ${ }^{3-11}$. In an especially remarkable series of studies, stretching over a period of some 10 years, Marusic, Mathis, Hutchins and their collaborators ${ }^{12-20}$ have investigated, mostly with hot-wire techniques, the response of the near-wall streaks in the viscosity-affected sublayer to the outer structures, the latter typically present at a distance of $0.1-0.2$ of the boundary-layer thickness from the wall. They show, in particular, that the outer structures affect the near-wall turbulent fluctuations in two ways: by "footprinting" and "modulation". The former is a process by which the outer large-scale motions impart correspondingly large translational fluctuations close to the wall, with some streamwise lag between the two - in essence, resulting in a near-wall field that is a superposition of large-scale footprints and the locally generated small-scale motions. The latter is a more subtle phenomenon, wherein the large-scale motions amplify or attenuate the small-scale turbulence depending on the sign of the large-scale footprints. The statistical characterisation of the correlation between the large-scale motion and the modulation is a contentious issue, however, and the subject of several recent studies $^{14,15,21,22}$.

Of particular relevance to the present study are the measurements and related analysis of footprinting and modulation by Mathis et $\mathrm{al}^{23}$ and Marusic et $\mathrm{al}^{17}$, following from earlier work by Mathis et $\mathrm{al}^{15}$, that has led to the proposal of an empirical relationship which "predicts" the effects of the large-scale outer fluctuations on the small-scale near-wall motions:

$$
u_{p}^{+}\left(y^{+}\right)=u^{*}\left(y^{+}\right)\left\{1+\beta\left(y^{+}\right) u_{O, L S}^{+}\left(y_{O}^{+}, \theta_{L S}\right)\right\}+\alpha\left(y^{+}\right) u_{O, L S}^{+}\left(y_{O}^{+}, \theta_{L S}\right)
$$

This relationship, in which (importantly) scaling is effected with the mean-friction velocity, contains two contributions, one including the coefficient $\alpha$ and the other $\beta$, that express, respectively, the effects of footprinting and modulation by the outer motions $u_{O, L S}^{+}\left(y_{O}^{+}\right)$on the canonical field $u^{*}\left(y^{+}\right)$that would exist if there were no large-scale structures, and $\theta_{L S}$ is the angle linking the outer motions with their footprints (reflecting a lag). The empirical coefficients $\alpha$ and $\beta$ depend on the wall distances $y^{+}$and $y_{O}^{+}$, but are of order 0.7 and 0.04, respectively, outside the viscous sublayer, varying only modestly beyond $y^{+} \approx 20$. The main concept underpinning eq. 1 is, therefore, that the near-wall intensity can be "predicted", at any Reynolds number and irrespective of the intensity of the outer motion, by imparting empirical corrections to the universal field $u^{*}\left(y^{+}\right)$that involve the Reynolds- 


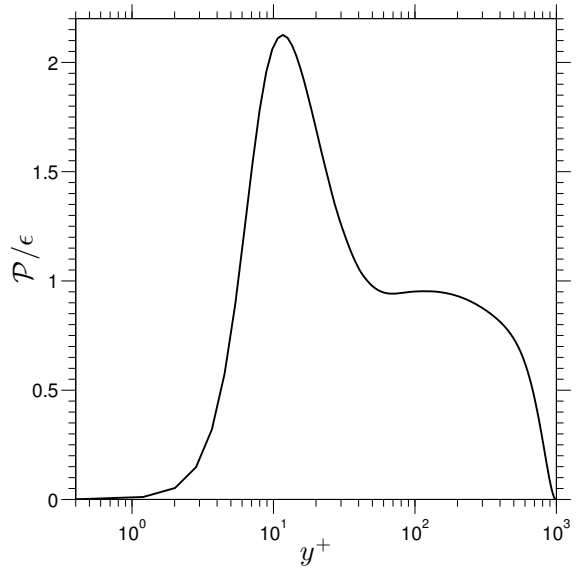

(a)

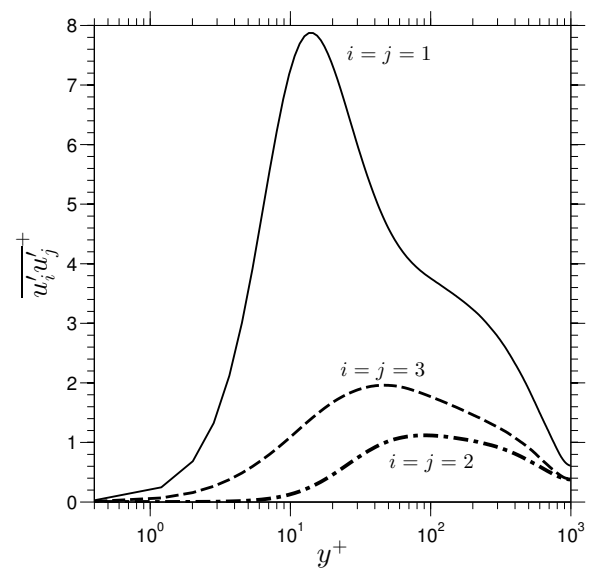

(b)

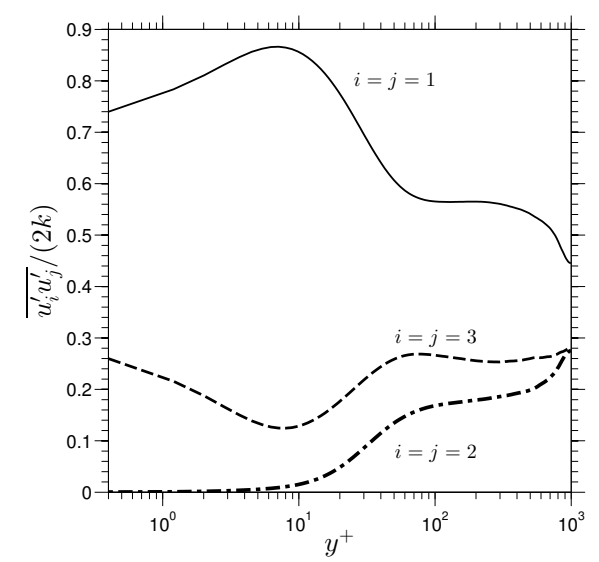

(c)

FIG. 1. Statistical evidence of large-scale outer structures: (a) ratio of turbulence-energy production to dissipation; (b) normal Reynolds stresses; (c) anisotropy of the normal Reynolds stresses.

number-dependent outer motions. On the assumption that the outer field $u_{O, L S}^{+}$is known, the predictive capability of eq. 1 thus hinges on the knowledge of $u^{*}\left(y^{+}\right)$, the empirical coefficients $\alpha$ and $\beta$ and the lag angle $\theta_{L S}$.

Eq.1 has two important implications that are open to question:

(i) positive and negative large-scale fluctuations cause equally-weighted modifications to the small-scale fluctuations - i.e. the unperturbed field $u^{*}$ is "symmetrically" altered;

(ii) scaling of all quantities (and thus also coefficients $\alpha$ and $\beta$ ) with the mean-friction velocity implies linearity, in the sense that the Reynolds-number universality expressed by eq. 1 is not conditional on large-scale variations in the friction velocity induced by the LS motions or the energy of the outer structures.

The latter restriction, in particular, has led Chernyshenko et $\mathrm{al}^{24}$ to re-examine the arguments underpinning eq. 1. The key point of difference lies in the proposal that universality is secured only if scaling is based on the local, large-scales-modified, friction velocity. Subject to the assumption of relatively weak large-scale fluctuations, permitting the linearization of assumed universal profiles of mean velocity and mean-square fluctuations, Chernyshenko et 
$\mathrm{al}^{24}$ derive coefficients $\alpha$ and $\beta$ corresponding to those in eq. 1 and having a magnitude and $y^{+}$-wise variations similar to those obtained experimentally by Mathis et $\mathrm{al}^{23}$.

In this paper, we examine various aspects of eq. 1, as well as the validity of Chernyshenko et al's proposal ${ }^{24}$, focusing on the statistical properties of the velocity fluctuations, which are derived from DNS data for channel flow at $R e_{\tau}=1025$, reported in Agostini et $\mathrm{al}^{25}$. Although this Reynolds number is relatively low - certainly in comparison with experimental configurations - the flow is appropriate to the aims pursued in this study. As is shown in Fig. 1(b), the streamwise turbulent stress is clearly elevated at $y^{+} \approx 100-400$, and there is an outer, albeit weak, maximum in turbulence-production-to-dissipation ratio, $\mathcal{P} / \epsilon$, around $y^{+} \approx 150$, corresponding to $y^{+} / h=0.15$. Significantly, Fig. $1(\mathrm{c})$ shows that this region is not simply characterized by a hump in the turbulence energy, but that the anisotropy experiences a significant elevation in the same region, showing a behaviour qualitatively similar to that at $y^{+} \approx 15$, and this provides an indication that the outer structures are akin to streaks, characterized by a preferential concentration of energy in the streamwise component. Finally, it will be shown in the discussion to follow that the pre-multiplied energy spectrum features a secondary outer (albeit weak) maximum that allows the outer scales to be separated from the inner ones.

Because the present examination is restricted to a single Reynolds number, it cannot shed light on the issue of Reynolds-number independence of eq. 1. Rather, the study aims to identify whether the response of the small near-wall scales to low-velocity and high-velocity large-scale outer motions is symmetric, whether - consistent with a symmetric response - a unique unperturbed field $u^{*}$ can be reconstructed from the DNS data and the coefficients provided by Mathis et $\mathrm{al}^{23}$ in eq. 1, and whether mean-friction scaling, or one based on the large-scales-modified local-friction velocity, is appropriate. In the final part of the paper, an alternative predictive model to eq. 1 is proposed, which provides an arguably superior phenomenological link between the small-scale and large-scale fluctuations, at least for the conditions studied.

Footprinting and modulation are of particular interest in the context of drag-reduction by periodic spanwise wall motion. At an actuation period close to an optimum value, at which the drag-reduction margin is maximised, the near-wall streaks are weak, and the effect of the large-scale structures on the near-wall streaks is very prominent, even at low Reynolds numbers. This is demonstrated by Touber \& Leschziner ${ }^{26}$ and Agostini et al ${ }^{25}$ for $R e_{\tau}=500$ and 1025, respectively. In particular, there is clear evidence of near-wallstreak modulation that leads to a virtual annihilation of the streaks in patches subjected to low-velocity footprints during periods of high rates of change in the spanwise Stokes-strain. It is observed, from a range of channel-flow DNS studies, that the drag-reduction margin declines approximately with $R e^{-0.2}$ - significantly faster than the rate proposed by the simple analysis of Luchini ${ }^{27}$ - and an important question is whether this is due to the increasing impact of the outer large-scale structures on the near-wall processes. This provides a major motivation for the present study.

\section{FLOW CONDITIONS AND SIMULATION DETAILS}

Results presented herein arise from a DNS for a canonical channel flow at $R e_{\tau}=1025$, performed over a box of length, height and depth $4 \pi h \times 2 h \times 2 \pi h$, respectively, corresponding to approximately $12 \times 2 \times 6 \times 10^{3}$ wall units. The box was covered by $1056 \times 528 \times 1056\left(=589 \times 10^{6}\right)$ nodes. The corresponding cell dimensions were $\Delta x^{+}$, 
$\Delta y^{+}{ }_{\min }, \Delta y^{+}{ }_{\max }, \Delta z^{+}=12.2,0.4,7.2,6.1$. The maximum CFL number was limited to 0.25. The computational scheme used is based on a co-located finite-volume method, with forth-order spatial discretisation, an explicit fractional time-stepping method, a third-order Gear scheme for time advancement, and a pressure-Poisson solver operating in conjunction with a multigrid scheme. The adequacy of the resolution and convergence of phase-averaged quantities were investigated in various ways, including a simulation of the flow over a grid of $1200 \mathrm{M}$ cells, an examination of the resolved dissipation, relative to the imbalance of other terms in the turbulence-energy budget, an evaluation of the ratio of cell distances to the Kolmogorov length scale, and comparisons of the turbulence energy budget to results by Moser et $\mathrm{al}^{28}$ and Hoyas and Jimenez ${ }^{29}$. As is demonstrated by Agostini et al ${ }^{25}$, the present budget agrees closely with the previously published data for the near-wall layer, within which resolution quality is the most critical. Moreover, Agostini et al ${ }^{25}$ show that the ratio of the cell volume $\left(\Delta x^{+} \times \Delta y^{+} \times \Delta z^{+}\right)^{1 / 3}$ to the Kolmogorov length scale, $\eta$, is close to 2 over the entire flow, with the $x$-directed ratio $\Delta x / \eta$ - the highest of the three directional ratios - being around 4 at $y / h=0.15$ and declining with $y$. Hence, the resolution in the outer part of the boundary layer is as good as, if not better than, in the near-wall layer.

\section{PROCESSING METHODOLOGY - SCALE SEPARATION}

The work of Marusic and his collaborators - eq. 1, in particular - is based on the processing of temporal signals over large periods of time, recorded at specific spatial wallnormal locations, over a range of wall-normal distances. This is not possible to replicate with the DNS data available in the present work, simply because the duration of the simulation is too short to enable a valid statistical representation of the effects of the outer structures. Instead, attention is focused on full-volume snapshots at a number of time levels.

A representative (raw) $x-z$ snapshot at $y^{+}=13.5$ is given in Fig. 2(a). This shows contours of streamwise-velocity fluctuations. The plot conveys a clear view of both the smallscale streaks, which are at maximum strength at the wall-normal location chosen, and of the footprints of large-scale outer structures, which typically have a length $x^{+}=\mathcal{O}\left(5 \times 10^{3}\right)$ or 5 channel half-heights for the present Reynolds number.

Given snapshots of the form shown in Fig. 2(a), the footprints need to be separated from the small-scale motions. This can be done in a variety of ways: Fourier decomposition with filtering, POD or some other mode-decomposition method. In this work, a method called "Huang-Hilbert Empirical Mode Decomposition" (EMD) has been favoured (not to be confused with Hilbert transform). Its main elements are summarised in Appendix A, in which reference is made to a number of signal-processing studies that investigates its properties and efficacy. In essence, the EMD is an algorithm that produces physically meaningful modal representations of data derived from arbitrary non-stationary or spatially varying processes, including amplitude- and frequency-modulated 1 -d and 2-d signals. The EMD splits any signal into a set of Intrinsic Mode Functions (IMFs) based purely on the local characteristic time/space scales of the signal. The method requires no pre-determined functional elements, such as Fourier or wavelet functions. Rather, the IMFs are the EMDgenerated basis functions, which arise purely from the given signal itself. Unlike Fourier methods, the EMD does not require filters to separate the scales, and does involve filterinduced loss of energy. POD is not well suited to the present conditions, because the energy content of the large scales is comparable to that of the small scales. The EMD was 


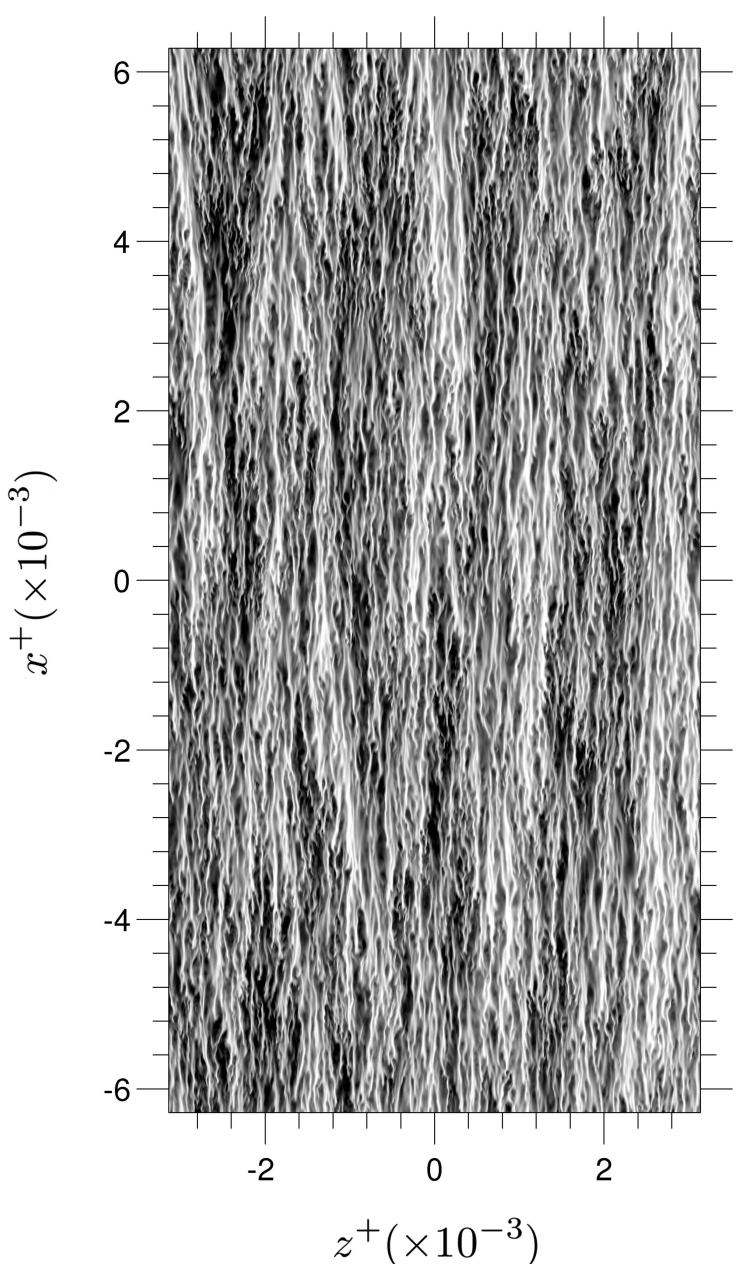

(a)

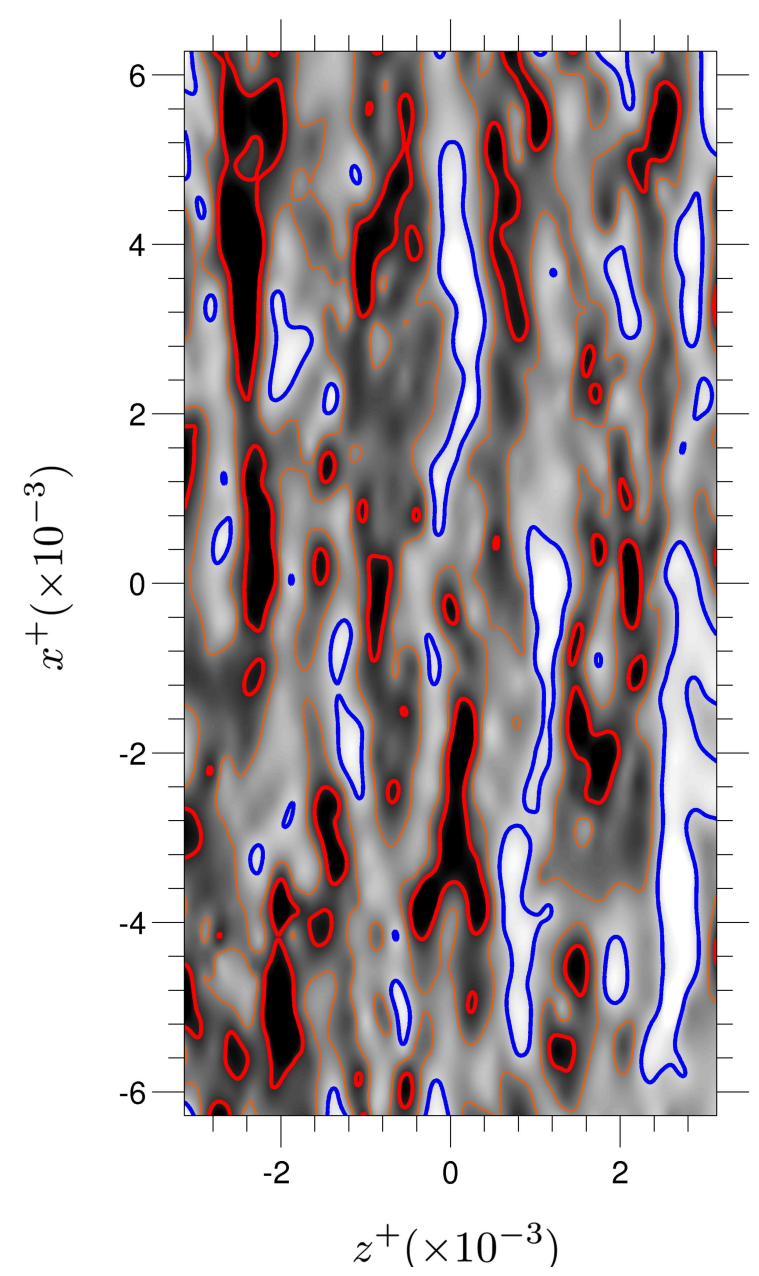

(b)

FIG. 2. Snapshot of the streamwise-velocity fluctuations at $y^{+}=13.5$ : (a) complete signal; (b) large-scale velocity fluctuations; islands with red/blue boundaries identify positive/negative fluctuations within the extreme $10 \%$ bands (tails) of the PDF of the large-scale fluctuations (see Fig. 7(b) and related description).

originally designed for 1-d signals. Its extension to 2-d fields is outlined in Appendix A, and has been investigated in several studies noted in the Appendix. The application of the EMD to snapshots of the form of Fig. 2(a) leads to the representation shown in Fig. 2(b) for the large-scale streamwise-velocity fluctuations, in which the islands surrounded by the line contours are areas within which the large-scale motions exceed a certain limit defined and discussed below. Typically, the large-scale velocity fluctuations within these islands are around $15-20 \%$ of the mean velocity (at $y^{+}=13.5$ ). Analogous large-scale fields may be obtained for the spanwise- and wall-normal-velocity components.

In principle, the number of IMFs determined from a raw signal is, within limits, a matter of choice, and this choice dictates, here, which modes are interpreted as representing, respectively, the small-scale and large-scale components of the full field. In the present case, snapshots of the form shown in Fig. 2(a) were decomposed into four modes, the first three representing the small scales and the last the large scales. This choice is justified on the basis of a preliminary analysis, the main results of which are presented in Figs. 3-6. 
Fig. 3 shows contour maps of pre-multiplied energy spectra, $k_{x} \Phi_{u u}^{+}$, of the streamwise fluctuations across $y^{+}$, presented in three different ways. All have been obtained by analysing 60 snapshots of the form shown in Fig. 2(a). Fig. 3(a) and Fig. 3(b) were both derived from the raw fields. The difference between the two is that the spectra in Fig. 3(b) are normalized forms of the those in Fig. 3(a), such that the area under any spectrum at any location $y^{+}$is 1 . This normalisation accentuates the dominant modes in different parts of the wall-normal domain. The implication is that the near-wall layer is dominated by small-scale ("SS", henceforth) modes of streamwise length scale $\lambda_{x}^{+}=\mathcal{O}(1000)$, while the outer region, at $y^{+} \approx 150$, is dominated by large scales ("LS", henceforth), $\lambda_{x}^{+} \approx \mathcal{O}(8000)$. When the raw signal is decomposed into four modes and pre-multiplied energy spectra are obtained, separately, for the sum of the first three modes and mode 4, the resulting two sets of contour maps are those given in Fig. 3(c). This figure thus illustrates that the LS modes are clearly delineated and closely related to the contour maps in Fig. 3(a). Moreover, the contours of the mode 4 are seen to penetrate deeply into the viscous sublayer, reflecting the pronounced footprinting of the LS structures observed in this layer. If, instead of applying the EMD to yield Fig. 3(c), energy spectra are derived from the raw fluctuation field, subject to lowpass and high-pass filters with cut-off at $\lambda_{x}^{+}=3000$, the resulting spectra feature respective small-scale and large-scale contour fields which are close to those shown in Fig. 3(c).

Next, the $y^{+}$-wise energy distributions of the SS and LS modes (modes 1-3 and mode 4, respectively) are determined and compared in Fig. 4 to distributions reported by Marusic et $\mathrm{al}^{17}$ for friction-Reynolds-number values of 3900, 7300 and 19000. The present SS energy is seen to agree well with the "universal", Reynolds-number-independent distributions of Marusic et $\mathrm{al}^{17}$. However, the energy of the LS motions is strongly Reynolds-numberdependent, and the present level is thus significantly lower than those corresponding to the higher Reynolds-number values. However, the level is broadly consistent with the trend suggested by the experimental data.

The validity of the LS energy in Fig. 4 extracted from mode 4 can be investigated by reference to the following empirical relations provided by Marusic et al ${ }^{17}$ for the variations of the total energy at $y^{+} \approx 15$ and $y_{O}^{+} \approx 3.9 \sqrt{R e_{\tau}}$, the latter regarded as the position of maximum energy of the large scales:

$$
\begin{aligned}
& <\left(u^{+}\right)^{2}>_{\text {inner peak }}=0.39 . \ln R e_{\tau}+4.8, \text { at } y^{+} \approx 15 \\
& <\left(u^{+}\right)^{2}>_{\text {outer peak }}=0.69 . \ln R e_{\tau}-0.85, \text { at } y_{O}^{+} \approx 3.9 \sqrt{R e_{\tau}}
\end{aligned}
$$

As is observed from Fig. 4, the SS energy is essentially Reynolds-number independent. It can thus be used to estimate the LS energy by subtraction from the energy levels given by eqs. 2 and 3 , namely:

$$
\begin{aligned}
<\left(u_{L S}^{+}\right)^{2}>_{\text {inner peak }} & =0.39 . \ln R e_{\tau}+4.8-\underbrace{[6]}_{\text {SS intensity }}, \text { at } y^{+} \approx 15 \\
<\left(u_{L S}^{+}\right)^{2}>_{\text {outer peak }} & =0.69 . \ln R e_{\tau}-0.85-\underbrace{\left[-0.69 . \ln \left(3.9 \sqrt{R e_{\tau}}\right)+6\right]}_{\text {SS intensity }}, \\
& =1.035 . \ln R e_{\tau}-5.91, \quad \text { at } y_{O}^{+} \approx 3.9 \sqrt{R e_{\tau}}
\end{aligned}
$$

The result is shown in Fig. 4(b) by means of the circles and stars, and this lends further 


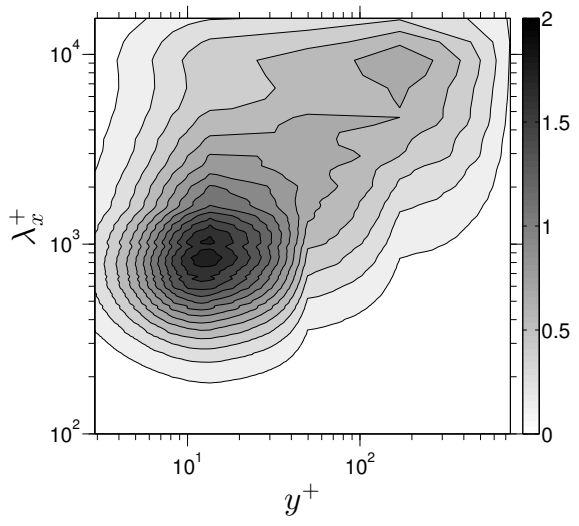

(a)

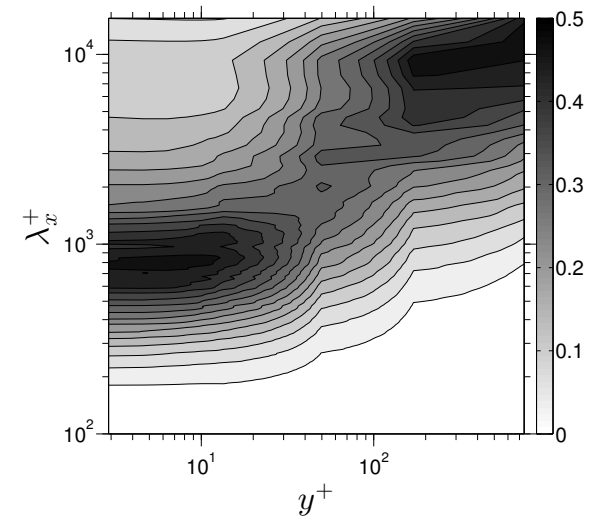

(b)

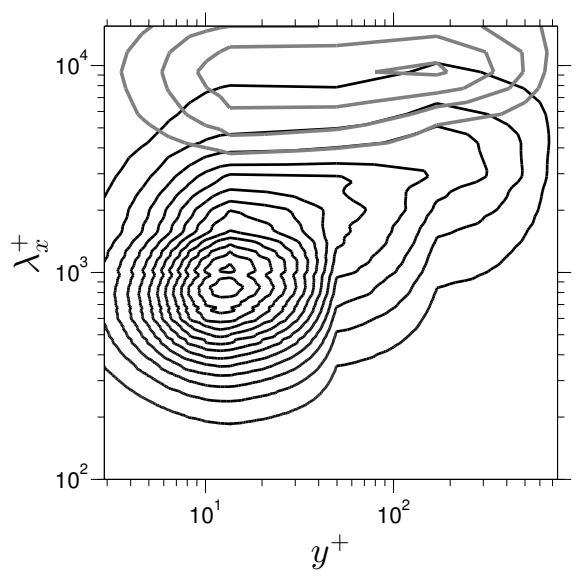

(c)

FIG. 3. Contours of pre-multiplied energy spectra $k_{x} \Phi_{u u}^{+}$for streamwise-velocity fluctuations : (a) standard (unscaled) representation; (b) scaled field, subject to normalisation $\left(k_{x} \Phi_{u u}^{+}\left(y^{+}\right)\right) /\left(\overline{u^{\prime} u^{\prime}}+\left(y^{+}\right)\right)$; (c) contours of pre-multiplied (unscaled) spectra for the LS mode (gray lines) and SS mode (black lines). Contours are separated by the constant interval 0.13, with lowest value being 0.13 .

support to the validity of the present scale-separation process. It has to be acknowledged, however, that uncertainties about the separation process increase with decreasing Reynolds number: at the present Reynolds number (but also at $R e_{\tau}=3900$ ), the large- and small-scale components feature comparable energy levels within most of the outer region.

Additional arguments that pertain to the LS and SS separation arise from the two-point correlation maps in Figs. 5 and 6. With attention focusing on the LS component, the correlation of the fluctuations at any $y^{+}$location, relative to those at a reference location $y_{O}^{+}\left(y_{O}^{+} \approx 150\right.$ in Fig. 5) is given by:

$$
C\left(y, y_{O}, \Delta x\right)=\frac{1}{N_{t} \times N_{z}} \sum_{n=1, k=1}^{N_{t}, N_{z}}\left(\frac{r_{12}\left(y, y_{O}, \Delta x, z_{k}, t^{n}\right)}{\sqrt{r_{11}\left(y, \Delta x, z_{k}, t^{n}\right)} \cdot \sqrt{r_{22}\left(y_{O L}, \Delta x, z_{k}, t^{n}\right)}}\right)
$$

wherein, 


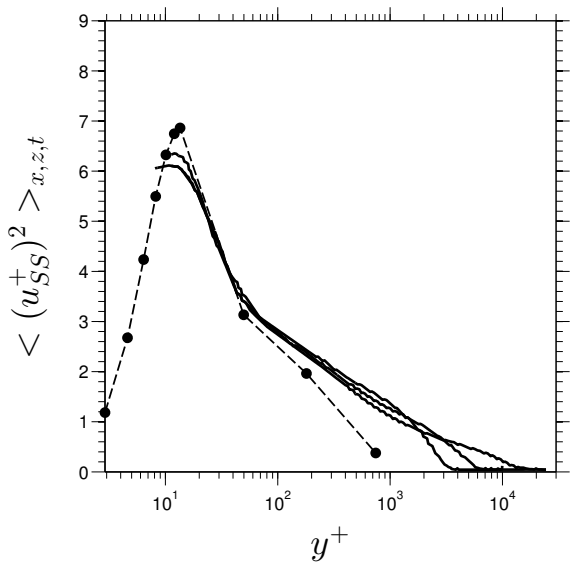

(a)

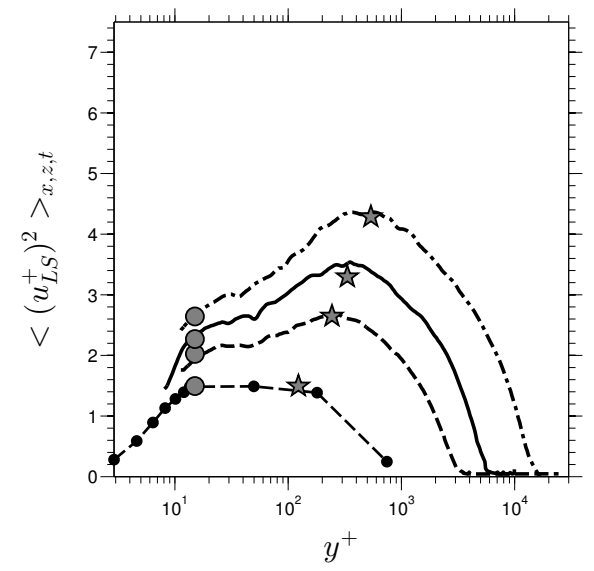

(b)

FIG. 4. Profiles of streamwise turbulence intensity profile: (a) SS scales, (b) LS scales; present results for $R e_{\tau}=1025$ : dashed lines and full circles; other profiles: experimental results of Marusic et $\mathrm{al}^{17}$ for $R e_{\tau}=3900,7300,19000$; stars identify the predicted wall-normal locations at which the maximum LS energy is expected, given by $y_{O}^{+}=3.9 \sqrt{R e_{\tau}}$.

$$
\begin{aligned}
r_{12}\left(y, y_{O}, \Delta x, z_{k}, t^{n}\right)= & \sum_{i=1}^{N_{x}}\left[u_{L S}\left(x_{i}+\Delta x, y, z_{k}, t^{n}\right)-<u_{L S}\left(y, z_{k}, t^{n}\right)>_{x}\right] \\
\times\left[u_{L S}\left(x_{i}, y_{O}, z_{k}, t^{n}\right)-<u_{L S}\left(y_{O}, z_{k}, t^{n}\right)>_{x}\right] & \\
r_{11}\left(y, \Delta x, z_{k}, t^{n}\right)= & \sum_{i=1}^{N_{x}}\left[u_{L S}\left(x_{i}+\Delta x, y, z_{k}, t^{n}\right)-<u_{L S}\left(y, z_{k}, t^{n}\right)>_{x}\right]^{2} \\
r_{22}\left(y_{O}, \Delta x, z_{k}, t^{n}\right)= & \sum_{i=1}^{N_{x}}\left[u_{L S}\left(x_{i}, y_{O}, z_{k}, t^{n}\right)-<u_{L S}\left(y_{O}, z_{k}, t^{n}\right)>_{x}\right]^{2}
\end{aligned}
$$

In eq. $6, \Delta x$ is the chosen separation distance in the streamwise direction between the $u_{L S}$ signals at $y_{O}$ and $y$ to be correlated, $N_{x}$ is the number of $x$-locations contributing to the correlation, with $x_{i}+\Delta x$ constrained to fall within the $x$-range of data available, $N_{z}$ and $N_{t}$ are, respectively, the total number of $z_{k}$ planes and $t^{n}$ levels contributing to the correlation, and $y_{O}$ is the $y$-location in the outer region - here, $y_{O}^{+} \approx 150$. In the case of the SS fluctuations, modes 1-3, the velocity $u_{L S}$ in eq. 6 has to be replaced by $u_{S S}$.

Fig. 5 shows a high degree of $y^{+}$-wise coherence in the LS motions, characteristic of footprinting, with the peak correlation level of approximately 0.5 down to the viscous sublayer. In contrast, Fig. 6 indicates that SS fluctuations in the viscous sublayer (relative to $y^{+}=15$ ), are hardly correlated with those in outer region (relative to $y_{O}^{+}=150$ ), while they are strongly correlated within the viscous sublayer itself. This decorrelation between the near-wall and outer regions supports the proposition that EMD modes 1-3 indeed characterize the SS motions.

In order to extract statistical data pertinent to the interactions between the large outer and small inner scales, and thus to eq. 1, 1-d and 2-d (joint) PDFs of SS velocity fluctuations have been assembled, conditional on regions of high-velocity, low-velocity and near-zero LS 


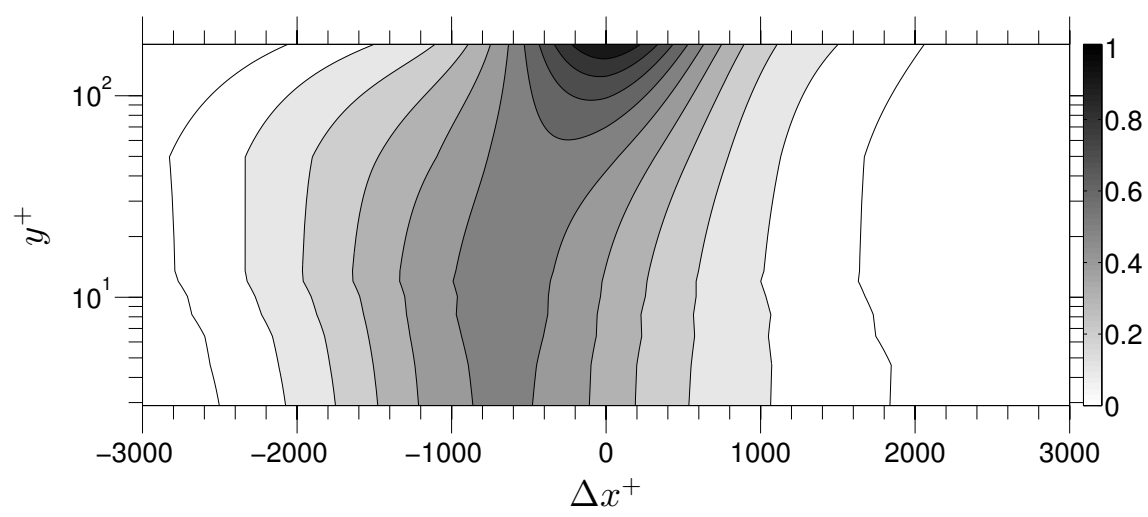

FIG. 5. Correlation of LS fluctuations at any $y^{+}$location with those at $y_{O}^{+} \approx 150$ (eq. 7).

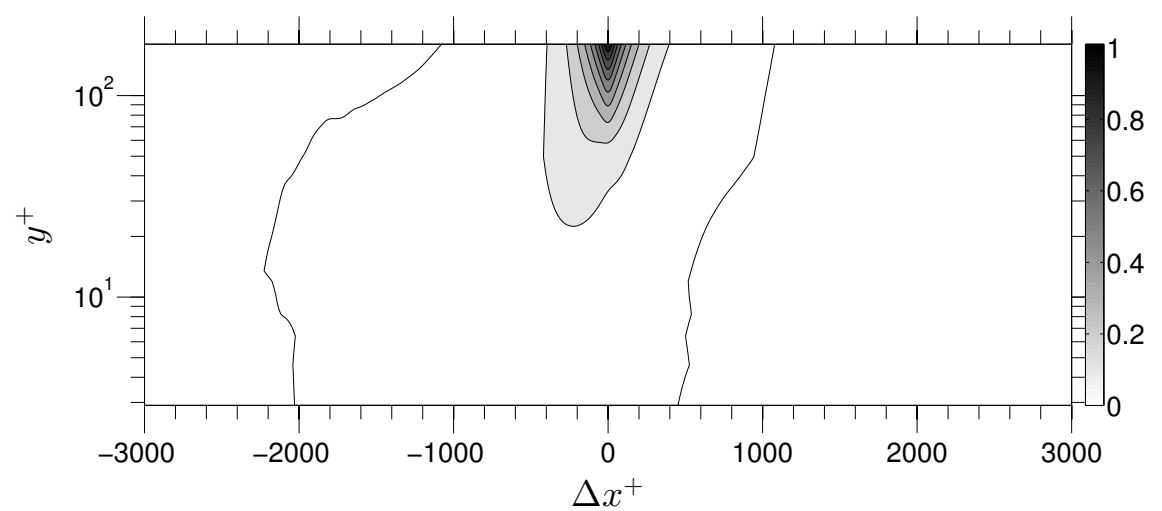

(a)

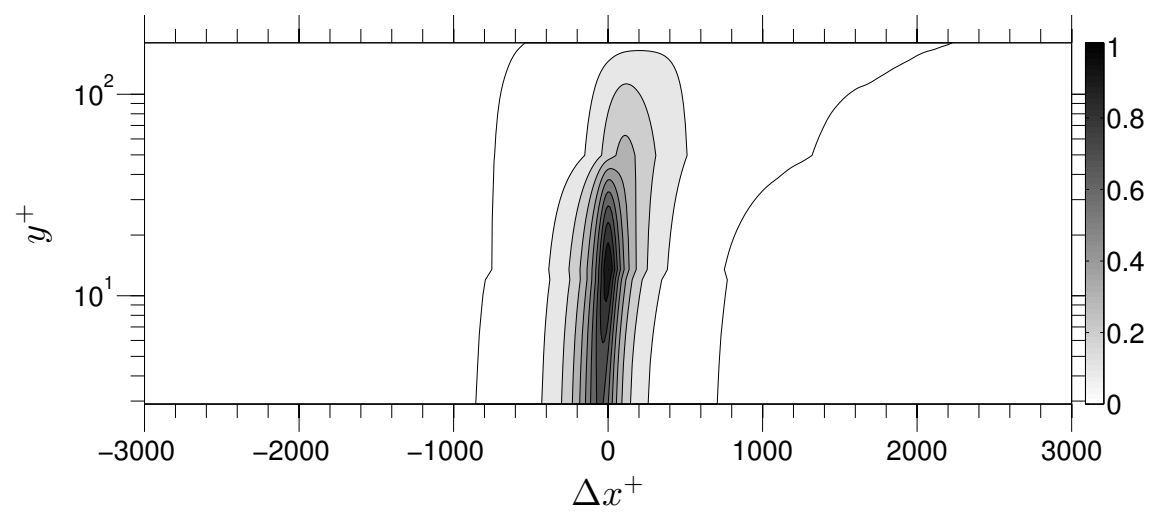

(b)

FIG. 6. Correlation of SS fluctuations at any $y^{+}$location with those obtained : (a) at $y_{O}^{+} \approx 150$ and (b) at $y^{+} \approx 15$

footprints. Regions of high-velocity and low-velocity LS motions are defined here as those which fall into the lowest and highest $10 \%$ bands of the PDF of the entire LS field, while regions which are, essentially, devoid of LS footprints are defined as those which fall into the central $10 \%$ of the PDF. This is illustrated in Fig. 7. The PDF in Fig. 7(a) gives the distribution of all LS motions contained in Fig. 2(b), while the two PDFs in Fig. 7(b) relate, respectively, to the top $10 \%$ (in terms of area) of positive (red) and negative (blue) 
LS fluctuations in the complete PDF of Fig. 7(a). These correspond, respectively, to the red and blue islands in Fig. 2(b), and LS fluctuations therein are of order $20 \%$ and 15\%, respectively, of the mean velocity in the same plane. It needs to be emphasized that there is no fundamentally profound reason for the present focus on $10 \%$ bands within the LS PDF. This is a choice that reflect the wish to bring out as clearly as is possible differences in the effects of positive and negative LS fluctuations on the SS motion. An analysis based on extended bands (up to 40\%) in no way changes, qualitatively, the conclusions presented herein.

With the extreme 10\% regions so identified, PDFs are then constructed of the SS motions within the blue and red regions, so to enable an examination of the effects of the footprints on the SS motions. A feature of the PDF in Fig. 7(a), which will be relevant to the discussion to follow, is that it is only weakly asymmetric, with extreme positive fluctuations slightly more prevalent than extreme negative ones. This weak asymmetry (relative to the principal axes) also applies to the joint $(u-v) \mathrm{PDF}$ at the same wall-normal location, shown in Fig. $7(\mathrm{c})$ and assembled with $v_{L S}^{+}$determined from the application of the EMD to the wall-normal component. The near-symmetry of the PDF in Fig. 7(c) will later serve as a background against which to contrast corresponding PDFs for the SS motions, which display a much higher degree of asymmetry.

\section{EXAMINATION OF SMALL-SCALE RESPONSE}

\section{IV.1. Interactions with positive vs. negative large-scale fluctuations}

Most results presented below are in the form of PDFs at the wall-normal location $y^{+}=$ 13.5, with mean-friction scaling of the velocity fluctuations. As such, they pertain to the "linear" model of Mathis et $\mathrm{al}^{19}$, eq.1, in which mean-friction scaling is also used. To investigate the issues addressed by Chernyshenko et $\mathrm{al}^{24}$, the sensitivity of the statistics to variations in the local friction velocity, $u_{\tau, L S}$, due to LS footprinting, are investigated, indirectly, by particular manipulations (as explained below) of PDFs at different wall-normal distances within a $y$-slab which would contains the surface of a $y^{+}=$constant when $y$ is scaled with the local friction velocity. To distinguish the two scaling options, the latter is identified by $y_{L S}^{+}$in the following discussion.

Some basic arguments are first conveyed by means of 1-d PDFs of the form $p d f\left(u^{+}\right)$. Figs. $8(\mathrm{a})$ - (c) give, respectively, PDFs of the total $u$-fluctuation field, of the SS-fluctuation field - i.e. with the LS motions subtracted from those of Fig. 8(a), and of the SS fluctuations normalized, for reasons explained below, with the absolute LS velocity as follows:

$$
u_{S S / L S}^{+}=u_{S S}^{+} \times \frac{<U_{1, L S}>_{x, z, t}}{u_{1, L S}+<U_{1, L S}>_{x, z, t}}
$$

where $\left\langle U_{1, L S}>_{x, z, t}\right.$ is the average velocity at $y^{+}=13.5$. Each plot contains three PDFs, relating to the lowest, middle and highest $10 \%$ bands, respectively, in the PDF of the large-scale motions shown in Fig. 7(a). Unless stated explicitly otherwise, normalisation is performed with the mean-friction velocity. The data forming the basis of these plots were collected from 602 -d snapshots spanning $t^{+}=t u_{\tau}^{2} / \nu=1000$. However, an examination of the PDFs for several individual snapshots, covering the range $t^{+}=1000$, shows very similar characteristics to the PDFs in Fig. 8. 


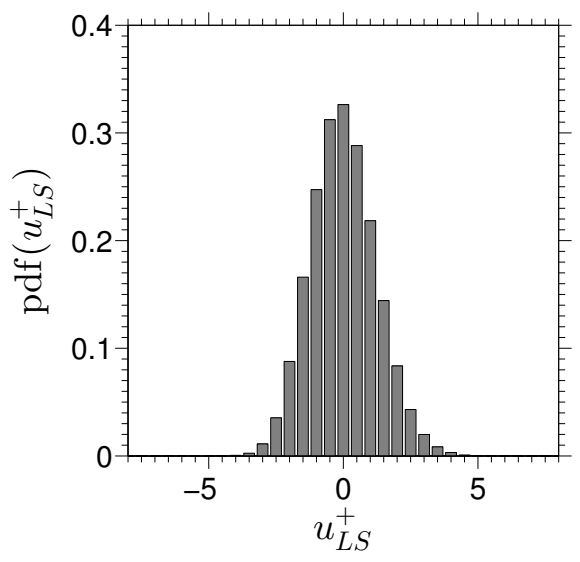

(a)

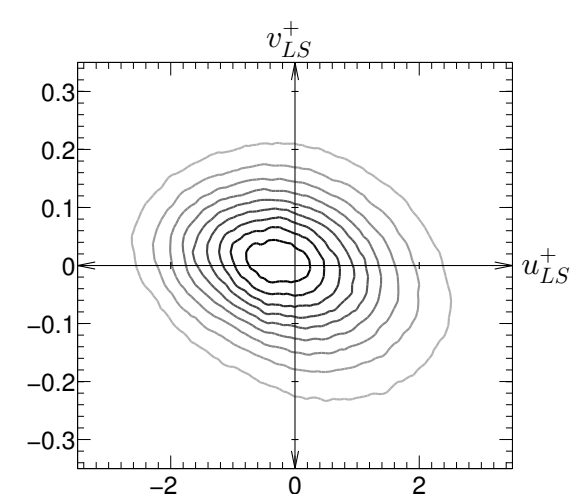

(c)

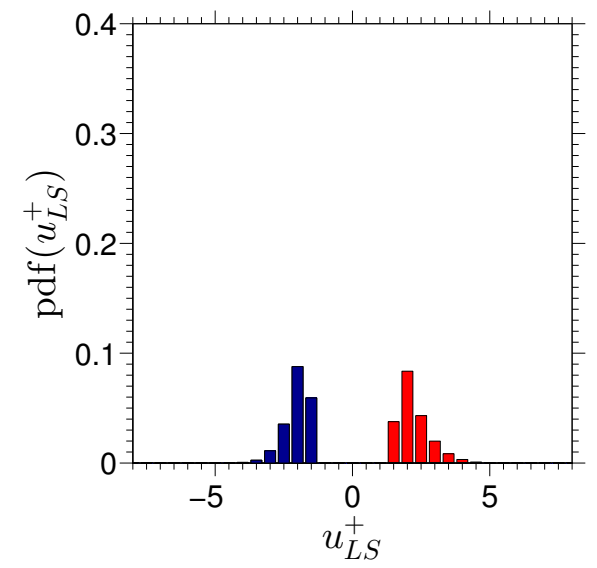

(b)

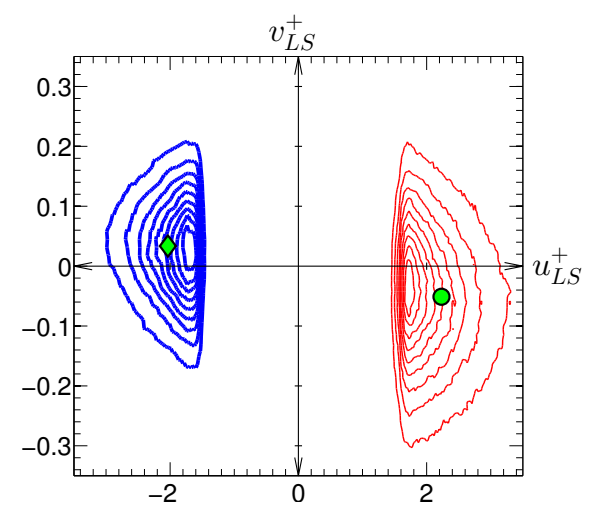

(d)

FIG. 7. PDFs of large-scale $u$-velocity fluctuations (EMD mode 4 ) at $y^{+}=13.5$ : (a) the whole 1-d PDF; (b) partial PDFs for the 10\% lowest and highest velocity fluctuations for which the statistics of SS motions are studied; (c) joint 2-d $(u-v)$ PDF of large-scale velocity fluctuations equivalent to (a); (d) partial joint 2-d $(u-v)$ PDFs equivalent to (b). PDF contours identify 0.1-0.9 of the PDF height at constant increment 0.1 .

This first comparison brings to light substantial differences in the manner in which the positive and negative LS motions affect the SS fluctuations. Importantly, this asymmetric response is not associated with the superposition of the LS motions onto the SS fluctuations - an effect that is included in Fig. 8(a), but is excluded from (b). When the LS motion is removed, the SS PDF associated with negative LS fluctuations is close to Gaussian, while that associated with positive LS fluctuations is skewed, the latter characterized by a predominance of relatively weak positive fluctuations and relatively strong negative fluctuations. The removal of the LS motions corresponds, essentially, to the shift implied by the $\alpha$-related term in eq. 1, which reflects the assumption of a superposition process. Here, however, $\alpha=1$ (by implication), because the LS information is available, and so used, on the same plane as the SS field, rather than at $y_{O}^{+}$. The rationale of normalizing the SS fluctuations with the absolute LS velocity, as done in Fig. 8(c), is rooted in the observation that the velocity ratio in eq. 7 agrees closely with the normalized friction velocity $u_{\tau} / u_{\tau, L S}$, the latter evaluated by applying the EMD scale-decomposition to the plane $y^{+}=3$ and restricting attention to the $10 \%$ extreme bands of the LS fluctuations. The implication of Fig. 8(c) 


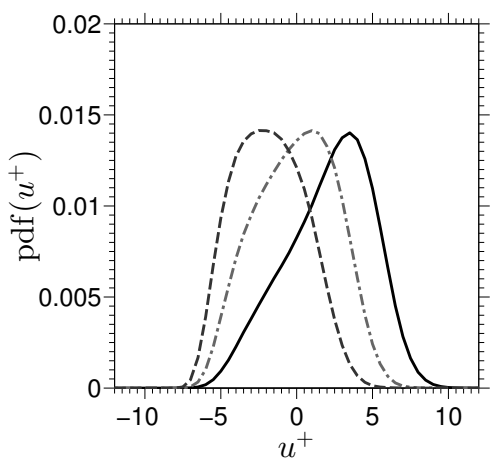

(a)

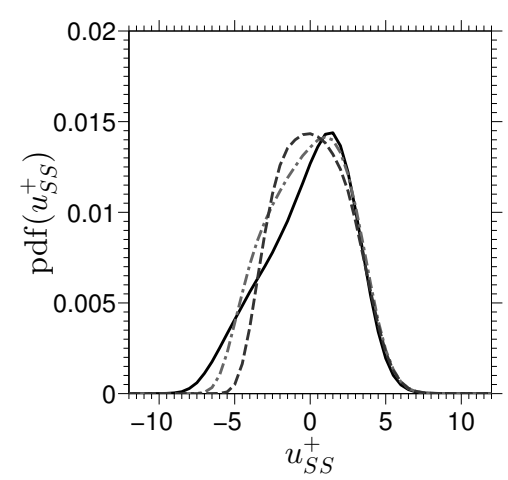

(b)

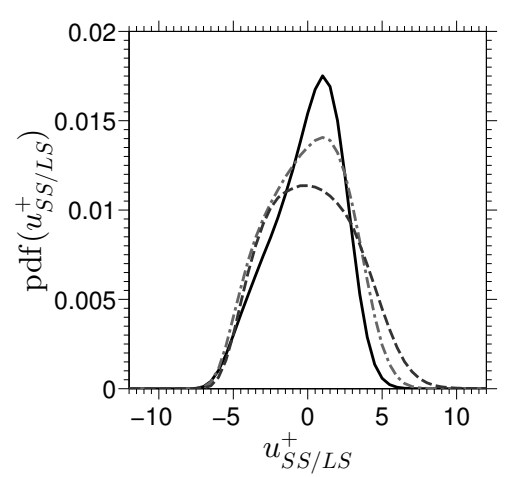

(c)

FIG. 8. PDFs of $u$-velocity fluctuations at $y^{+}=13.5$ pertaining to the bands of $10 \%$ extreme positive LS events (solid line), 10\% minimum LS events (chain line) and 10\% extreme negative events (dashed line); (a) total fluctuations; (b) SS fluctuations only; (c) SS fluctuations normalized with the LS velocity, eq. 7 .

is, therefore, that the SS fluctuations neither scale universally with $u_{\tau}$ nor with $u_{\tau, L S}$ when these fluctuations are determined or considered at a fixed $y^{+}$location. The fact that the modulation of the SS fluctuations differs markedly for positive and negative LS fluctuations, in contrast to the implication of eq. 1, will be argued below to reflect the impact of splatting associated with sweeps. As a consequence, the modulation is not simply representable in terms of the streamwise LS fluctuations alone.

The use by Mathis et $\mathrm{al}^{23}$ of $\theta_{L} \approx 12.5^{\circ}$ and $\alpha=O(0.7)$ in eq. 1 to correlate the LS motions near the wall to those at $y_{O}^{+}=3.9 \sqrt{R e_{\tau}}$ is examined in Figs. 5 and 9 (a), respectively, the former containing the correlation map for the LS motions, and the latter showing the $y^{+}$-wise variations of the standard deviation of the PDF for the streamwise LS motions, thus reflecting their intensity. The $y^{+}$-wise locus of the maximum correlation coefficient in Fig. 5 suggests $\theta_{L S} \approx 12.5^{\circ}$ for the straight line connecting the maxima at locations $y^{+}=15$ and $y_{O}^{+}=3.9 \sqrt{R e_{\tau}}$, while Fig. 9(a) shows that the intensity of the LS motions is fairly constant, down to the viscous sublayer within which the standard deviation drops progressively. The implication is that $\alpha=O(1)$ is more appropriate than 0.7 , at least for $y^{+}$above the viscous sublayer. Hoewever, this applies only to the present Reynolds number. As Fig. 4(b) and eqs. 4 and 5 show, $\alpha$ declines with increasing Reynolds number. The $y$-wise variations of the wall-normal and spanwise standard deviation of the PDFs of the LS motions - Fig. 9(b) and Fig. 9(c), respectively - will be required in Section 4.4 for a proposal that is an alternative to eq. 1, one that also allows the prediction of the spanwise near-wall SS fluctuations.

In eq. $1, u^{*}$ is assumed to be the canonical signal that would have been recorded in the absence of LS effects. In the present context of spatial statistics, it is reasonable to suppose that equivalent conditions prevail in areas to which the central portion of the LS PDF in Fig. 7(a) relates. Fig. 8 shows, by chain lines, the 1-d PDFs of the SS motions that relate to the central $10 \%$ band in the LS PDF. As might be expected, the SS fluctuation field in this central band has features intermediate between the those within the two extreme areas of the LS PDF of Fig. 7. However, this does not suffice, on its own, to judge this field as being free from any LS influences. The interpretation of the $u^{*}$ field as either being or not being "universal" is one aspect of an examination of the validity of Mathis et al's model ${ }^{23}$ by reference to joint $(u-v)$ PDFs, considered next. 


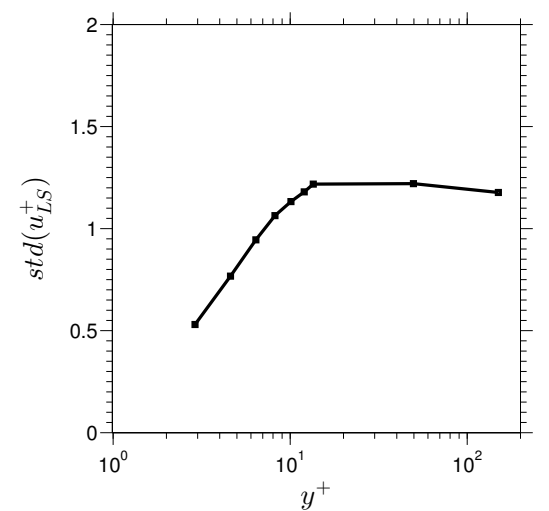

(a)

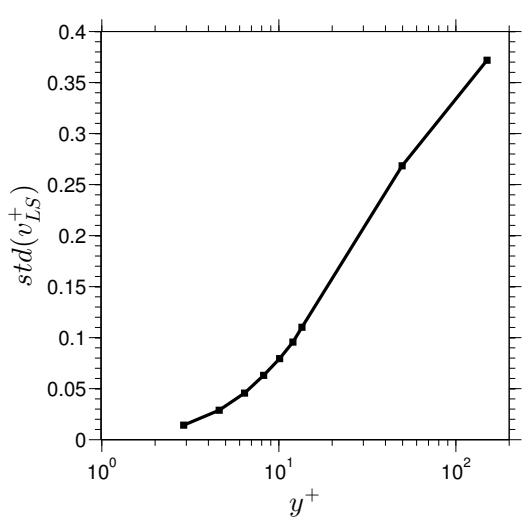

(b)

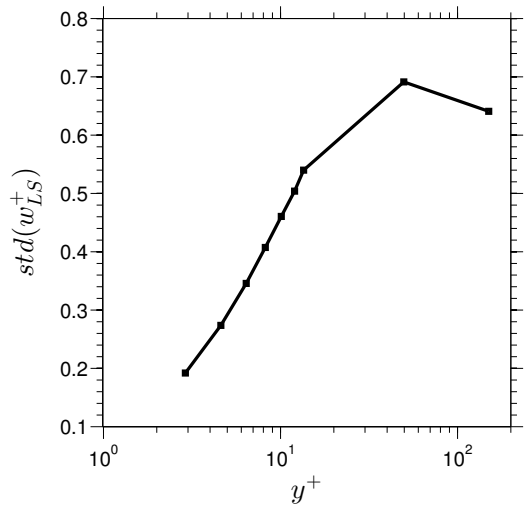

(c)

FIG. 9. $y$-wise variation of the standard deviation of the PDFs of the LS motions for : (a) streamwise velocity; (b) wall-normal velocity and (c) spanwise velocity.

Fig. 10 shows PDFs of the entire $(u-v)$-fluctuation field, of the total fluctuations in the blue and red areas of Fig. 2, of the SS fluctuations in the blue and red areas, and of the SS fluctuations scaled by the LS velocity, in accordance with eq. 7 , with $v_{S S / L S}^{+}$ (and later also $w_{S S / L S}^{+}$) obtained upon replacing $u_{S S}^{+}$by $v_{S S}^{+}$(or $w_{S S}^{+}$). The circular and diamond-shaped symbols represent the centres of gravity of the PDFs in question. The collapse of the centres of gravity in Fig. 10(c) points to the validity of the superposition component associated with $\alpha$ in eq. 1. There is, furthermore, clear evidence that positive LS perturbations cause considerably stronger modulation of the SS motions than negative perturbations. Once scaled by the LS velocity, Fig. 10(d), the SS fluctuations display fair universality in quadrant 2, associated with ejections, but substantial departures from universality in quadrant 4 . That these drastic differences are not associated with a bias in the LS motions is demonstrated in Fig. 7(c), which shows the near-symmetric PDF of the complete field of LS motions.

Fig. 11 gives joint $(u-w)$ PDFs, which correspond to the $(u-v)$ PDFs in Fig. 10 . These suggest that the skewed shape of the PDF in Fig. 8, associated with positive LS motions, is driven by sweeps which transport relatively weak SS fluctuations from beyond the buffer layer downwards. In contrast, SS ejections are weaker, more numerous and more normally distributed, thus unaffected by the bias caused by sweeps. In common with the $(u-v)$ PDFs, the centres of gravity of the $(u-w)$ PDFs collapse upon the removal of the LS motion. Similarly, the SS motions scaled by the LS velocity collapse for negative LS motions, but not for positive ones.

The conclusion thus emerging, so far, from the above discussion is that modulation is not a "symmetric" process, in the sense of positive and negative LS motions having the same weight on the SS field, and that the lack of symmetry is caused by major differences in the effects of sweeps and ejections on the SS structure. In particular, the $(u-w)$ PDFs bring to light that sweeping motions go hand in hand with strong spanwise fluctuations in quadrants 1 and 4, which are characteristic of splatting. This process, and its effects on the SS motions, is not accounted for in eq. 1 and cannot be captured by the model. 


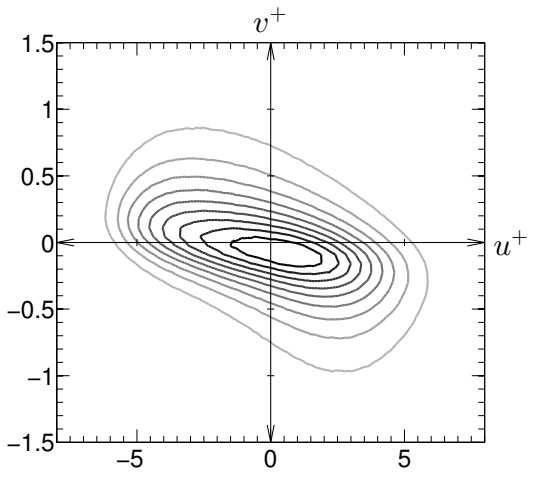

(a)

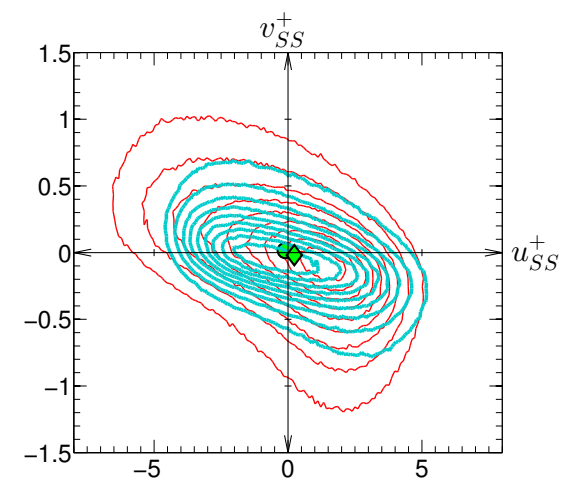

(c)

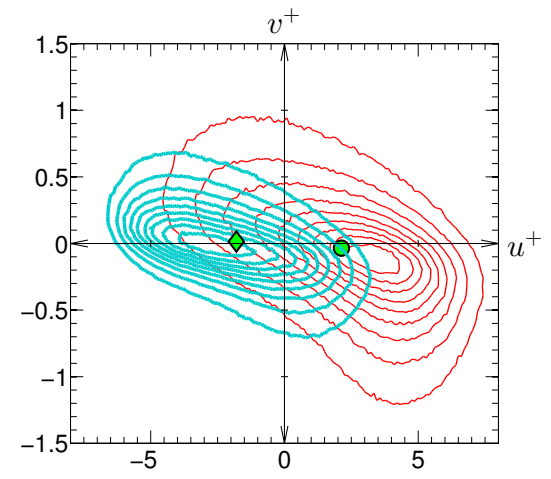

(b)

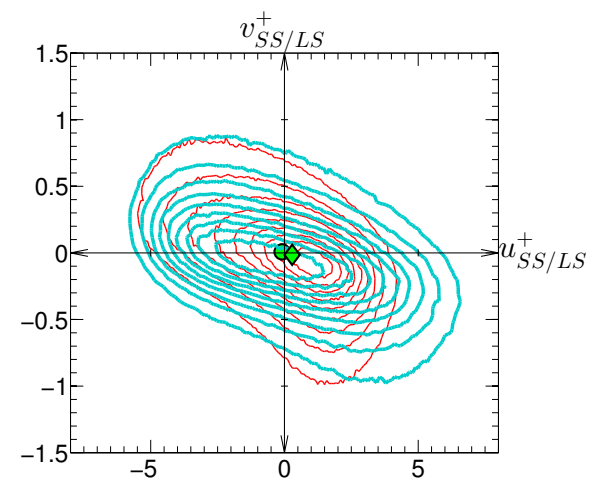

(d)

FIG. 10. Joint $(u-v)$ PDFs at $y^{+}=13.5$ of (a) total fluctuation field; (b) total fluctuations in blue and red regions in Fig. 2(b), respectively; (c) SS fluctuations; (d) SS fluctuations normalized by LS fluctuations. Red contours : $+10 \%$ LS fluctuations, blue contours $-10 \%$ LS fluctuations. PDF contours identify 0.1-0.9 of the PDF height at constant increment 0.1, subject to total PDF volume normalized to 1.

\section{IV.2. Universal SS motions - the $u^{*}$-field}

If, despite the above incompatibility, the model is to be retained, it is possible to determine (or rather estimate) the values for $\alpha$ and $\beta$ that secure the best possible compliance with the present data. To this end, eq. 1 is re-cast as follows:

$$
u^{*}=\frac{u^{+}-\alpha u_{O, L S}^{+}}{1+\beta u_{O, L S}^{+}}
$$

Given values $\alpha$ and $\beta$, the question is whether the SS PDFs, conditional on the $\pm 10 \%$ extrema of the LS motion, can be made to collapse, such a collapse being interpretable as representing the field $u^{*}$. An ambiguity that arises with this process relates to the interpretation of $v^{*}$ and $w^{*}$. In the absence of a credible alternative, the assumption is made here that $v^{*}=v_{S S}^{+}$and $w^{*}=w_{S S}^{+}$, respectively. A possible variation is to use eq. 8 , with the numerator replaced by $v_{S S}^{+}$and $w_{S S}^{+}$to obtain $v^{*}$ and $w^{*}$, respectively. However, this variation has only marginal effects on the results to follow.

As shown in Fig. 12 , use of $\beta=0.04, \alpha=0.7$, with $u_{O, L S}$ taken from $y_{O}^{+} \approx 150$, subject to $\theta_{L S}=12.5^{\circ}$, results in a fair correspondence in the $u$-wise widths of the PDFs, 


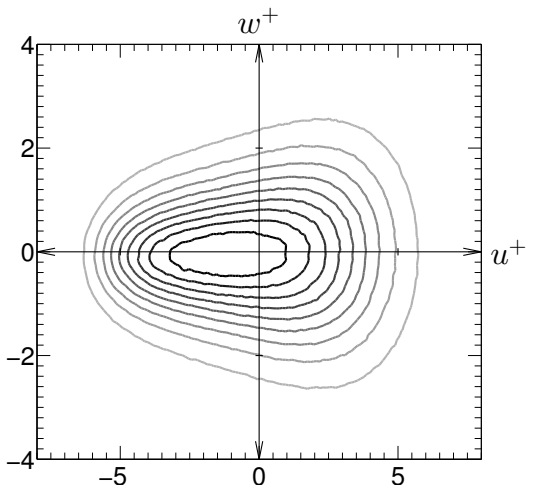

(a)

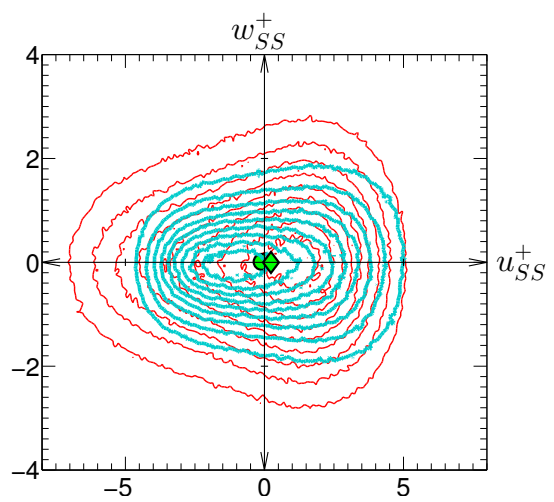

(c)

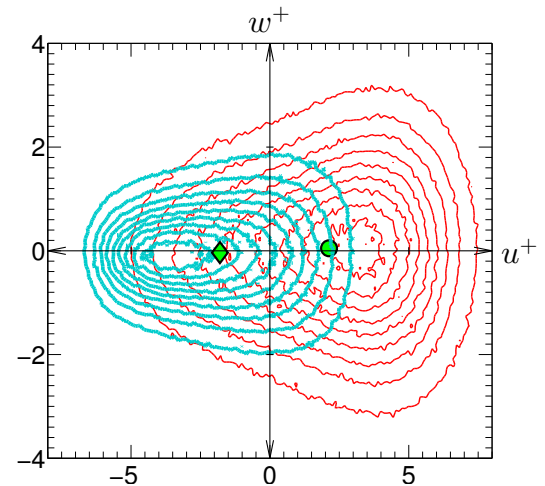

(b)

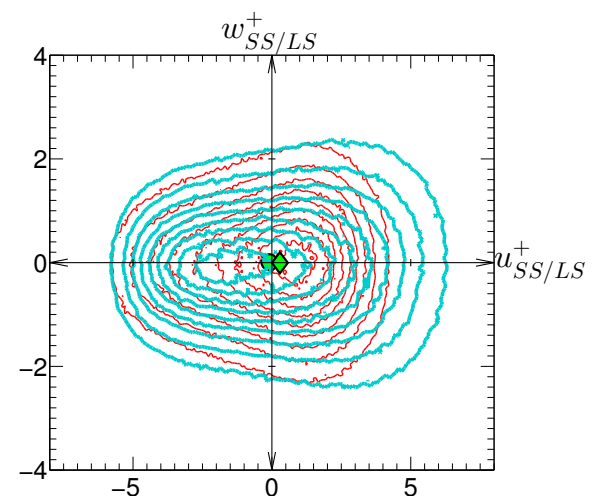

(d)

FIG. 11. Joint $(u-w)$ PDFs at $y^{+}=13.5$ of (a) total fluctuation field; (b) total fluctuations in blue and red regions, respectively in Fig. 2(b); (c) SS fluctuations; (d) SS fluctuations normalized by LS fluctuations. Contours levels: see caption of Fig. 10.

but in significant differences in their shape, especially in quadrant 4. Thus, although Fig. 12 suggests that the amplitude, or intensity, of the $u^{*}$-fluctuations is fairly insensitive to whether the LS motions are positive or negative, supporting Mathis et al's model (with the particular empirical constant $\beta$ used), the PDFs in Fig. 12 show that $u^{*}$-field is not, in fact, universal, because the PDF of the SS fluctuations subjected to positive LS fluctuations is skewed and distorted (see also Fig. 8(b)). Moreover, if the joint $\left(u^{*}-v^{*}\right)$ PDFs are projected onto the $u^{*}$ axis, to yield corresponding 1-d PDFs, the shape of the latter are quit similar to that shown in Fig. 8(c). This distortion is, again, a consequence of the sweeping motions - an effect that does not fall neatly under the heading "footprinting" and amplitude "modulation", and which cannot be represented purely by reference to the outer LS motions $u_{O, L S}^{+}$.

\section{IV.3. Effects of local-friction scaling}

The question as to whether scaling by the local LS-modified friction velocity, rather than the mean value, is conducive to the "prediction" of a universal $u^{*}$ field, as suggested by Chernyshenko et $\mathrm{al}^{24}$, is not easy to answer unambiguously within the present processing strategy. Strictly, the requirement is to construct PDFs on $y^{+}=$constant (i.e. $y_{L S}^{+}$) surfaces, 


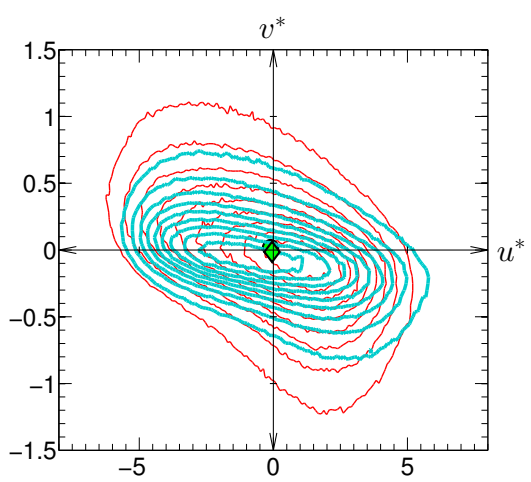

(a)

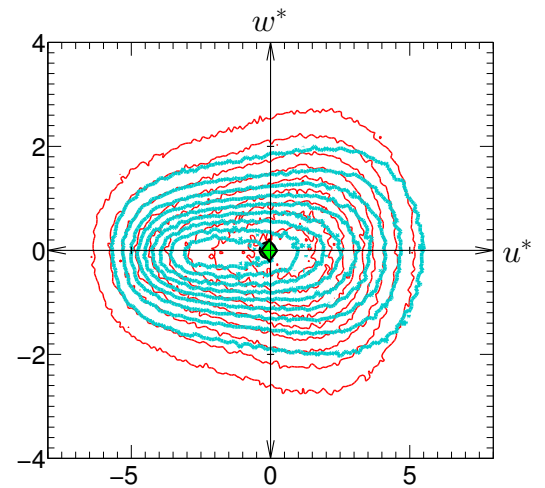

(b)

FIG. 12. Joint PDFs: (a) $u^{*}-v^{*}$; (b) $u^{*}-w^{*}$, both derived from eq. 8 at $y^{+}=13.5$, with $\beta=0.04$ and $\alpha=0.7$. Contours levels: see caption of Fig. 10 .

subject to scaling with the local $u_{\tau, L S}$. A more straightforward, albeit somewhat pragmatic, two-pronged approach adopted here is as follows.

As noted earlier, there is a close correspondence, in the regions corresponding to the extreme $\pm 10 \%$ bands of the PDF of the LS motions, between the values of the LS-velocity fluctuations, normalized by the absolute LS velocity at $y^{+}=13.5$, and LS fluctuations in the friction velocity, normalized by the mean value. Moreover, the intensity of the LS motions hardly varies in the range $y^{+}=10-150$. This correspondence between the fluctuations in the LS velocity and the associated friction velocity is exploited in the present examination.

In the first approach, the conditions on the planes $y^{+}=10$ and 13.5, based on meanfriction scaling, form the basis for estimating conditions on the plane $y^{+}=12$, based on local scaling. To this end, the regions corresponding to the LS events within the top $10 \%$ of the LS PDF for $y^{+}=10$ are identified, while for $y^{+}=13.5$, the bottom $10 \%$ of the LS events are determined. These regions, from both planes, should now all correspond fairly closely to $y^{+}=12$, based on local scaling; in other words, $y_{L S}^{+} \approx 12$. Importantly, the SS fluctuations within the above LS regions, which are examined here, are subject to local scaling, i.e. are normalized with $u_{\tau, L S}$. The PDFs for the SS fluctuations determined for the two sets of regions at the two planes are shown in Fig. 13(a), the red contours arising from the highvelocity LS fluctuations at $y^{+}=10$ and the blue contours from low-velocity LS fluctuations at $y^{+}=13.5$. As is observed, there is a good level of collapse of the two PDFs, and this supports, prima facie, Chernyshenko's proposition of universality based on local LS scaling. Thus, either of the PDFs may be taken to represent the universal SS field $u^{*}$, and this field is a significant improvement over that shown in Fig. 12(a). It is also observed to be close to the $(u-v)$ PDF corresponding to that shown in Fig. 8(a), derived from regions of low LS motions.

The second approach again starts with planes $y^{+}=10$ and 13.5. For both planes, regions of LS fluctuations corresponding to $y_{L S}^{+}=12$ are here estimated by first identifying iso-lines of the locally-scaled absolute velocity $U^{+}=12$, i.e. scaled by $u_{\tau, L S}$. Any domain (strip) around the iso-line within which SS statistics are examined is then defined by the constraint that only the events in the LS PDF within $\pm 5 \%$ of $U^{+}=12$ may fall into the domain. The regions so identified are then assumed to correspond, approximately, to $y_{L S}^{+}=12$. The two PDFs for the SS motions, again normalized by $u_{\tau, L S}$, are shown in Fig. 13(b); red and blue contours relate, respectively, to the lower and upper $y^{+}$slices. The degree of collapse 


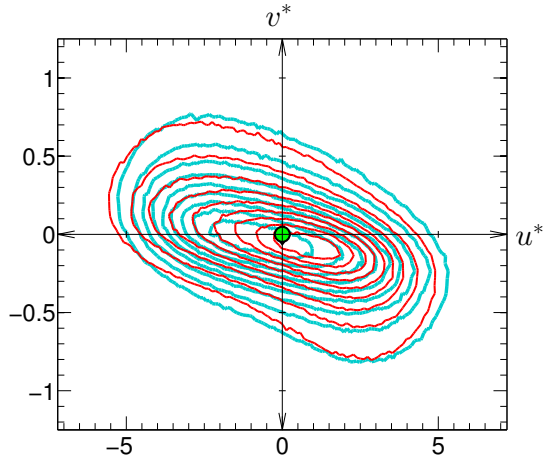

(a)

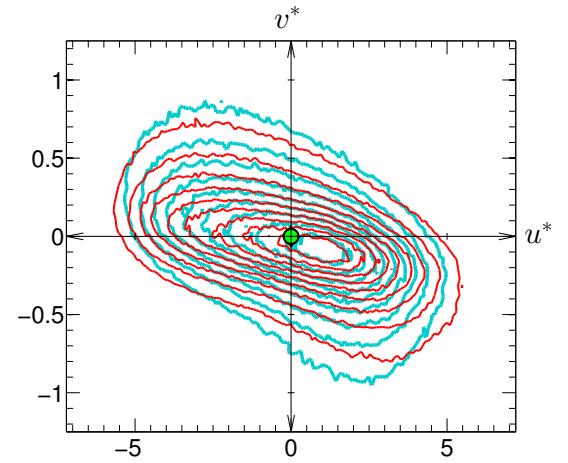

(b)

FIG. 13. Joint PDFs for SS motions at approximately constant $y^{+}=12$, extracted from the meanscaled slices $y^{+}=10$ and 13.5; (a) red contours identify SS motions conditional on top $10 \%$ of the LS motions at $y^{+}=10$; blue contours identify SS motions conditional on bottom $10 \%$ of the LS motions at $y^{+}=13.5$; (b) contours representing SS motions within regions in which $u_{L S}^{+}=12 \pm 5 \%$; red contours determined from $y^{+}=10$ plane, blue contours determined from $y^{+}=13.5$ plane. For contour levels: see caption of Fig. 10.

is clearly not as good as that shown in Fig. 13(a). The outcome of both methods must be viewed with caution, because of the assumptions and approximations made. The second method is, arguably, better founded, but the noisy contours reflect some scarcity of data or noisy definition of the LS-areas from which the SS statistics are derived. They do, however, provide some evidence in support of the proposition that universality of the SS motions may only be secured if scaling is effected with the local LS-modified friction velocity.

\section{IV.4. Alternative predictive model for universal SS motions}

In view of the above discussion, the question may be posed as to whether another, purely phenomenological, model can be derived that provides an improved representation of the interaction between the LS structures and the near-wall SS structures, via an improved description of the $u^{*}$ field. Such a model is proposed below.

Assuming the LS footprints are available at the near-wall location $y^{+}$under consideration, the following relationships are proposed to apply, so as to represent the dependence of the SS fluctuations, $u_{i, S S}^{+}(i=1,2,3)$, on the LS motions, $u_{L S}^{+}$, at one and the same plane:

$$
\begin{aligned}
u_{i, S S}^{+} & =\frac{U_{i}-\left(u_{i, L S}+<U_{i, L S}>_{x, z, t}\right)}{u_{\tau}} \\
u_{i, S S / L S}^{+} & =u_{i, S S}^{+} \times \frac{<U_{1, L S}>_{x, z, t}}{u_{1, L S}+<U_{1, L S}>_{x, z, t}} \\
u_{i}^{*} & =u_{i, S S / L S}^{+} f\left(U_{1, L S}, \chi_{i}\right)
\end{aligned}
$$

where 


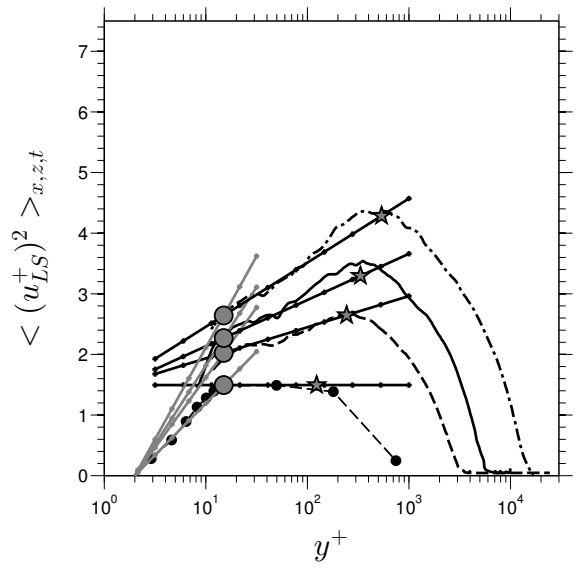

(a)

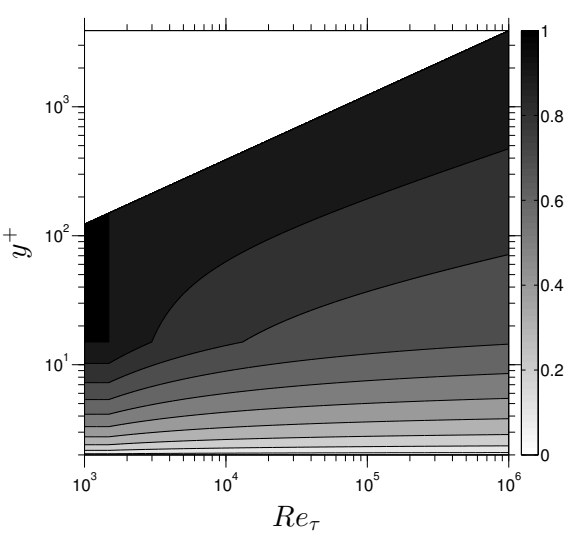

(b)

FIG. 14. Dependence of footprinting intensity on wall-normal distance and $R e_{\tau}$; (a) approximation of $y^{+}$-variations of LS energy, for key of lines, see Fig. 4; (b) variation $\alpha_{1}\left(y^{+}, R e_{\tau}\right)$ in eq. 12

$$
f\left(U_{1, L S}, \chi_{i}\right)=1+\chi_{i} \times \frac{u_{1, L S}}{<U_{1, L S}>x, z, t}, \chi_{i}=1 \text { if } i=1 \text { and } u_{1, S S}^{+}>0 \text {, otherwise } \chi_{i}=0 .
$$

In the above equations, it is important to distinguish between the LS fluctuation $u_{i, L S}$, the plane-parallel average of which is zero, and the absolute LS velocity, $U_{i, L S}$, and its finite plane-parallel average, $\left\langle U_{1, L S}\right\rangle_{x, z, t}$.

If, in contrast to $u_{L S}^{+}$, the only LS information available is $u_{i, O, L S}^{+}$at the outer location $y_{O}^{+}, u_{i, L S}^{+}$may be obtained from:

$$
u_{i, y^{+}, L S}^{+}(x)=\alpha_{i}\left(y^{+}, R e_{\tau}\right) \cdot u_{i, O, L S}^{+}\left(x+\Delta x\left(y^{+}\right)\right)
$$

where $\alpha_{i}=\operatorname{std}\left(u_{i, L S}^{+}\right) / \operatorname{std}\left(u_{i, O, L S}^{+}\right)$and are based on the distributions contained in Fig. 9. For example, in the case of the streamwise velocity, $u_{1, L S}^{+}$is estimated from the outerflow signal $\left(\alpha_{1} \times u_{1, O, L S}^{+}\right)$and replaced in equations (9)-(11). In accordance with Fig. 9(a),

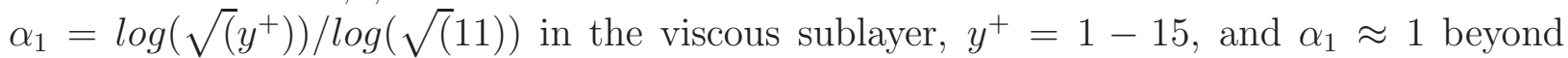
$y^{+}=15$. This uniformity is consistent with the constant portion $\left(u_{L S}^{+}\right)^{2}$ in Fig. 4(b). However, the data of Marusic et $\mathrm{al}^{17}$ for higher Reynolds numbers can be used to derive a more general variation of the form $\alpha_{1}\left(y^{+}, R e_{\tau}\right)$. To this end, the variations of $\left(u_{L S}^{+}\right)^{2}$ can be approximated by the straight lines shown in Fig. $14(\mathrm{a})$, i.e., $\left(u_{L S}^{+}\right)^{2}=A \cdot \ln \left(y^{+}\right)+B$. The resulting variation of $\alpha_{1}\left(y^{+}, R e_{\tau}\right)$ is shown in Fig. 14(b), and this demonstrates that $\alpha_{1}$ tends to decline as $R e_{\tau}$ increases, assuming a value around 0.7 at $R e_{\tau} \approx 40000$.

Finally, there remains the need to determine $\Delta x\left(y^{+}\right)$, representing the $x$-wise lag in the LS footprinting. With attention confined to the region $15 \leq y^{+} \leq y_{O, L S}^{+}=3.9 \sqrt{R e_{\tau}}$, Fig. 5 shows that the locus of maximum correlation of LS fluctuations is roughly a straight line with angle:

$$
\gamma=\tan ^{-1}\left[\frac{\ln \left(y^{+}\right)-\ln \left(y_{O, L S}^{+}\right)}{\Delta x^{+}\left(y^{+}\right)}\right] \approx 0.23^{\circ}
$$

Hence, the actual angle in real space, $\theta\left(y^{+}\right)$, varies with $y^{+}$. For the particular location, $y^{+} \approx 15, \Delta x^{+} \approx 610$ and $\theta(15)=\theta_{L S}$ in eq. 1 is approximately $12.5^{\circ}$. 


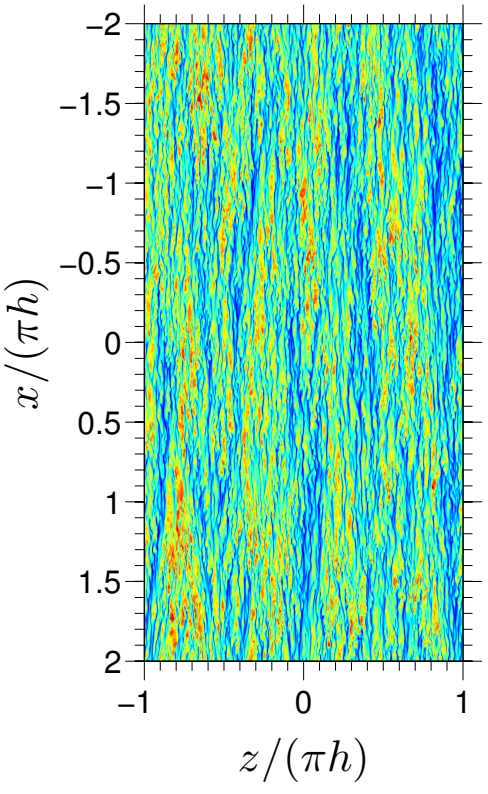

(a)

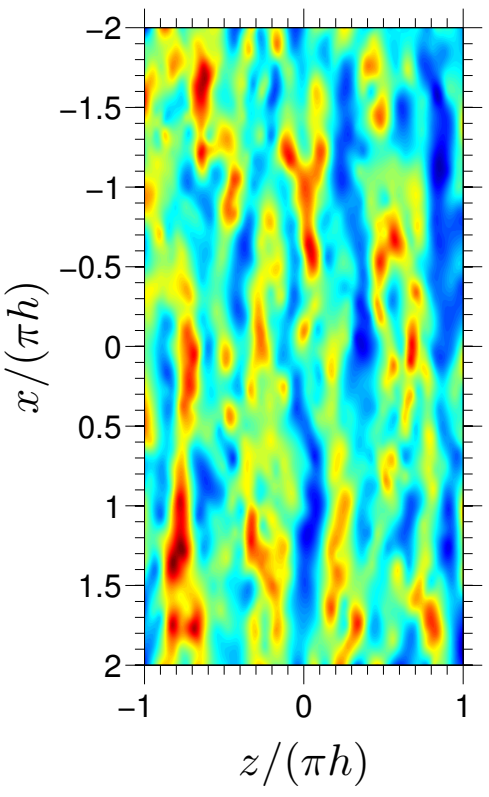

(b)

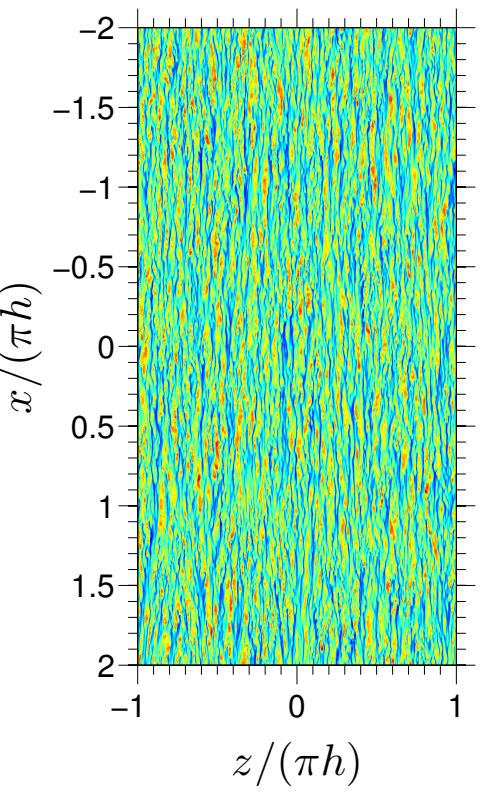

(c)

FIG. 15. Snapshot of streamwise-velocity fluctuations at $y^{+}=13.5$; (a) complete signal; (b) large-scale Motions; (c) $u^{*}$-field derived from eq. 11

It is not possible here to examine the fidelity of this model for any but the present Reynolds number $R e_{\tau}=1025$, and this done in Figs. 15 and 17. First, Fig. 15 provides a qualitative view of the $u^{*}$-field predicted by eq. 11 in comparison with the complete (raw) field at $y^{+}=13.5$ and the field of the LS motions. The only important point that this figure conveys is that the $u^{*}$-field shows no evidence of the LS motions. What this figure cannot show, however, is the presence or absence of modulation and any asymmetry therein. Such information is conveyed by Figs. 16-17 which show, respectively, joint $(u-v)$ and $(u-w)$ PDFs for $y^{+}=13.5$, both derived from eq. 11. Each figure compares three alternative proposals: in (a) are shown PDFs obtained directly from the DNS data in the $10 \%$ central portion of the LS PDF of Fig. 2(a), where the SS motions should be least altered by the LS motions; in (b) PDFs arising from eq. 8 (i.e. Mathis et al's model ${ }^{23}$ ) are reproduced; in (c) are shown PDFs obtained with the present proposal, with the LS fluctuations taken from the same level as that to which the PDF applies; and in (d) are shown the PDF constructed with the LS motions extracted from $y_{O}^{+}=150$, rescaled in accordance with Fig. 9 and subject to the $\Delta x^{+}$shift obtained from eq. 13.

The present model is observed to give a much improved level of collapse to that achieved with Mathis et al's model ${ }^{23}$ when the LS motions are taken from the same level at which the SS PDFs are considered. When the LS motions are taken from $y^{+}=150$, the level of collapse is slightly degraded. Apart from reducing the distortions in the $u^{*}$-field that arise from the different effects of the positive and negative LS fluctuations, the model also provides a creditable representation of the $w^{*}$-field. 


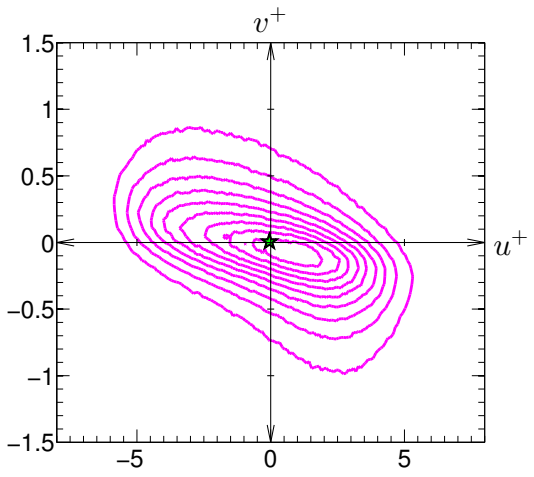

(a)

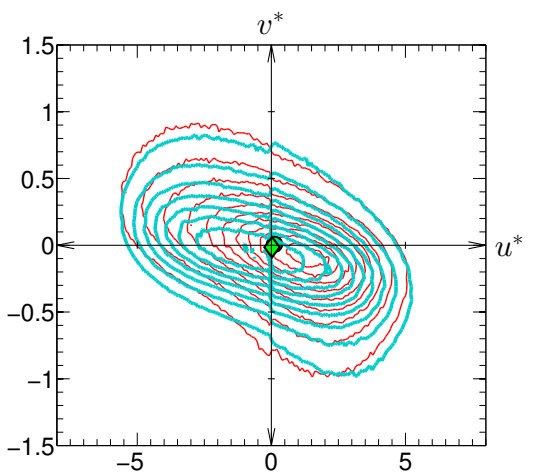

(c)

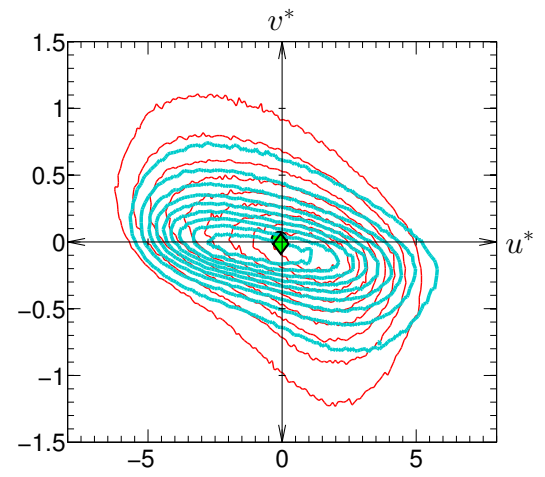

(b)

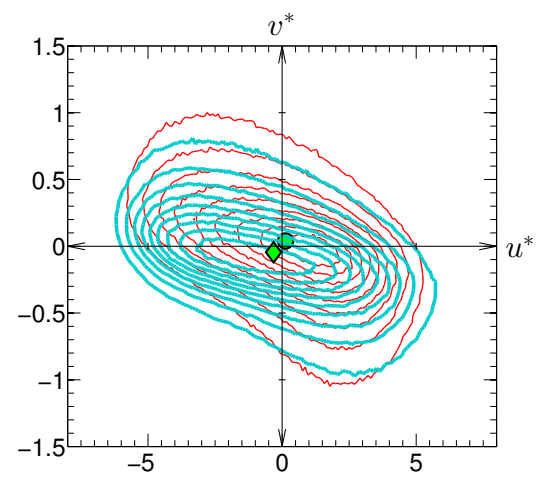

(d)

FIG. 16. Joint $u^{*}-v^{*}$ PDFs at $y^{+}=13.5$ : (a) from $10 \%$ middle band of LS PDF; (b) Mathis et al's ${ }^{23}$ model (eq. 8); (c) present model with LS motion taken at $y^{+}=13.5$; (d) present model with LS motion taken from $y^{+}=150$. Definition of contours: see caption of Fig. 10.

\section{SUMMARY AND CONCLUSIONS}

This study set out to examine, in general, the effects of large-scale motions in the log-law region on the small-scale streaks in the viscosity-affected near-wall layer, and to investigate, in particular, the validity of the concepts underpinning the predictive model of Mathis et al. ${ }^{23}$, which expresses these effects by way of superposition- and modulation-related terms acting on a "universal" small-scale field that is held to be unaffected by the large-scale motions. An additional objective was to examine whether a universal representation is aided by scaling with the local, large-scales-modified friction velocity, rather than the mean value.

In contrast to an analysis of time-signals at specific wall-normal locations, as performed with the experiments upon which the predictive model was based, the present approach is based on spatial statistics - mostly 1-d and 2-d PDFs - derived from spatial snapshots at 60 time levels spanning 1000 viscous time units. Nevertheless, it is argued that the spatial strategy is valid as a means of illuminating the relevant issues. A central element of the spatial analysis is the particular, non-standard, mode-decomposition method used to separate the large scales from the small scales, which does not rely on the imposition of pre-defined filters, and which is dictated purely by the scales contained within the signal itself. The resulting decomposition is justified extensively on the basis of pre-multiplied energy spectra, correlation maps and energy profiles for both scales, the last in comparison 


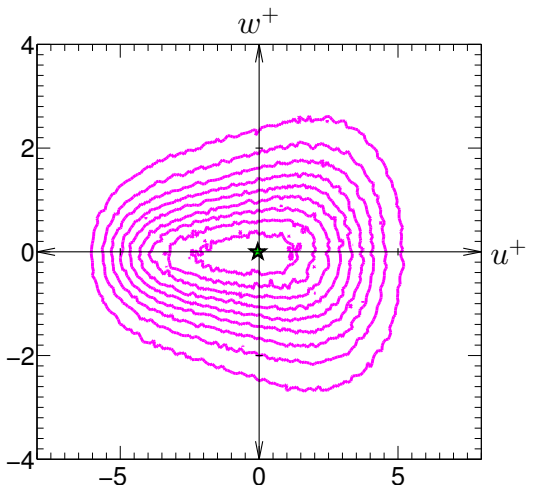

(a)

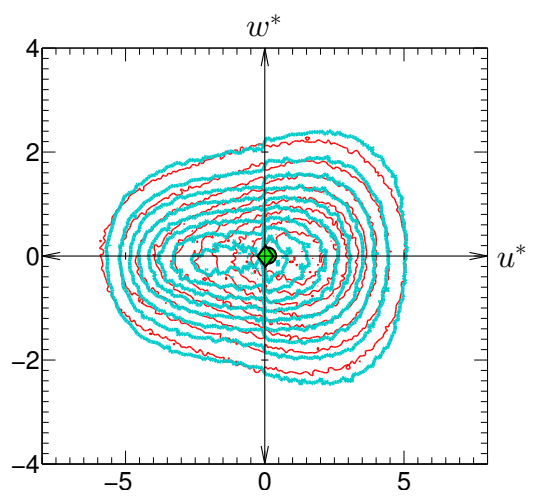

(c)

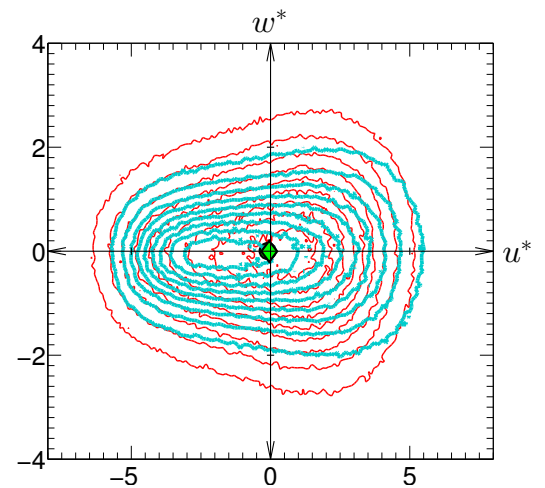

(b)

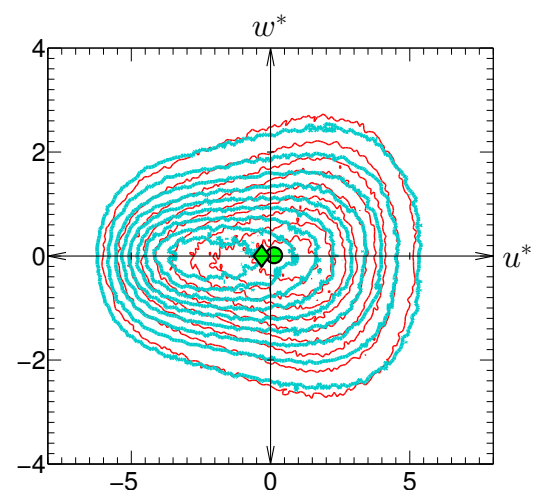

(d)

FIG. 17. Joint $u^{*}-w^{*}$ PDFs at $y^{+}=13.5$ : (a) from $10 \%$ middle band in LS PDF; (b) Mathis et al's model $^{23}$ (eq. 1); (c) present model with LS motion taken at $y^{+}=13.5$; (d) present model with LS motion taken from $y_{O}^{+}=150$. Definition of contours: see caption of Fig. 10.

of published experimental data at higher Reynolds numbers.

With scaling based on the mean-friction velocity, as done by Mathis et al. ${ }^{23}$, the results show that there are significant differences in the response of the small-scale motions to negative and positive large-scale outer fluctuations. In other words, the response of the small-scale motions is not "symmetric", in the sense of the use the $\beta$-term in the model of Mathis et al, which represents modulation. In reality, the small-scale streaks are modified in three ways, rather than two: by superposition, by modulation and by distortions of the small-scale field caused the differential influence of sweeps (splatting) and ejections (antisplatting). The third process is brought to light by distinctive distortions in both the $(u-v)$ and $(u-w)$ PDFs. The PDFs included herein, as well as others at lower $y^{+}$values not included, suggest that the differential influence of the sweeps and ejections of the large-scale motions is felt down to the lower levels of the viscous sublayer $\left(y^{+} \approx 3\right)$. In particular, the pear-shaped $(u-w)$ PDFs point to the presence of splatting that is associated with sweeps, which is argued to be the cause of the asymmetry in the modulation of the streamwise small-scale fluctuations.

While the model of Mathis et al. ${ }^{23}$ correctly represents the superposition effect exerted by the large-scale structures on the near-wall structures, it does not appear to capture correctly the asymmetric modulation, due to the splatting-induced distortions noted above. Furthermore, use of the model equations to extract the "universal" small-scale motions 
from the DNS data shows that these motions are not, in fact, "universal", insofar as these motions depend on whether they originate from (or associated with) regions of positive or from negative large-scale fluctuations. These observations are not, therefore, consistent with the model.

Two attempts have been made to investigate a modified model ${ }^{24}$, in which scaling is based on the local large-scale-modified friction velocity, rather than the mean value. The analysis, although admittedly only approximate, suggests that the inconsistencies highlighted above in the context of the linear model are weakened by the local scaling.

An alternative, purely phenomenological, predictive model has been proposed for the "universal" velocity fluctuations, held to be unaffected by the large-scale motions. In this model, superposition is represented by eq. 9, modulation by eq. 10 and the splattinginduced distortions by eq. 11. These yield the "universal" small-scale field without the use of the empirical coefficients of Mathis et al. ${ }^{23}$. The model also describes the influence of the large-scale motions on the wall-normal and spanwise small-scale field. Qualitative and quantitative results presented herein indicate that this model gives a modestly better representation of the "universal" field, relative to Mathis et al.'s model ${ }^{23}$. This is especially so when the large-scale motions that are used in the model are taken from the same plane as that at which the small-scale motions are determined. Use of the outer (log-layer) large-scale motions, instead of those at the plane in question, requires rescaling based on DNS-derived wall-normal variations of the standard deviations of PDFs of the large-scale motions, and this process tends to degrade the predictive accuracy of the model somewhat. Further model refinements are possible, but this is not a central element of the rationale of the present study. In any event, such improvements would require an analysis of DNS data at higher Reynolds numbers than the present value.

\section{ACKNOWLEDGMENTS}

The authors acknowledge the financial support provided by the UK academic funding agency EPSRC, through grant EP/G061556/1, EADS UK Limited and Airbus Operations Limited. The simulations were performed on the UK's National HPC facility HECToR, as part of the aforementioned EPSRC grant and with additional resources provided by the EPSRC-funded UK Turbulence Consortium. 


\section{APPENDIX A: OUTLINE OF EMPIRICAL MODE DECOMPOSITION METHOD}

The EMD is an algorithm proposed by Huang et $\mathrm{al}^{30}$, which can produce physically meaningful modal representations of data derived from arbitrary non-stationary or spatially varying processes, including amplitude- and frequency-modulated 1-d signal. The EMD splits any signal into a set of Intrinsic Mode Functions (IMFs) based purely on the local characteristic time/space scale of the signal. The method requires no pre-determined functional elements, such as Fourier or wavelet functions. Rather, the IMFs are the EMD-generated basis functions, which arise from the given signal itself.

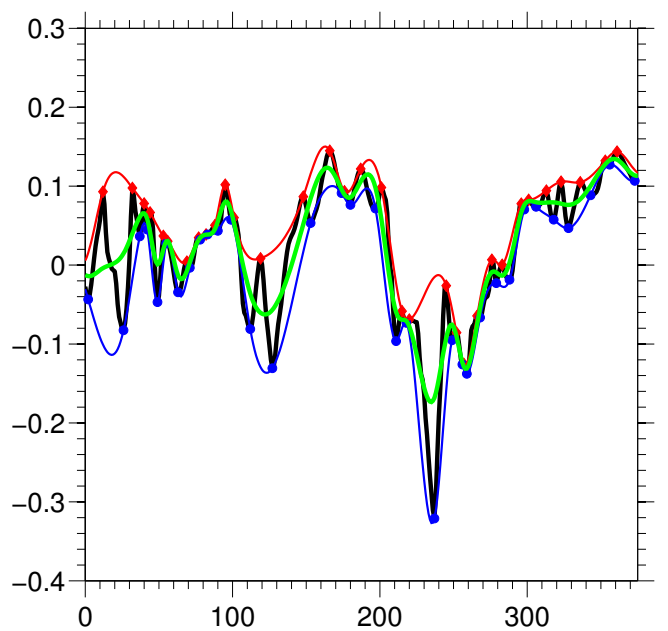

FIG. 18. Illustration of the principles of Huang et al's EMD algorithm, by reference to an arbitrary 1-d signal: upper and lower envelopes are defined by the local maxima and minima, respectively, and the mean value of the envelopes is given in green

The method is outlined first by reference to the time-dependent signal shown (identified as Data) in Fig. 18. This signal, denoted by $f(x)$ or $f(t)$, is now subjected to the following iterative process:

1. A residual $\operatorname{res}(x)$ is defined and initialised by $\operatorname{res}(x)=f(x)$.

2. The local minima and maxima of $\operatorname{res}(x)$ are identified, and upper and lower envelopes, $E_{u p}(x)$ and $E_{l o w}(x)$, respectively, are fitted to the maxima and minima. This is done with the aid of cubic-spline functions.

3. The mean of the two envelopes is computed: $E_{m n}(x)=\left(E_{u p}(x)+E_{l o w}(x)\right) / 2$

4. The mean envelope is subtracted from the residual: $\operatorname{res}(x) \leftarrow \operatorname{res}(x)-E_{m n}(x)$

5. The residual is subjected to a stopping criterion, based on the mean-squares difference between consecutive residuals, normalized by the square of the residual itself. If the criterion is satisfied then the first IMF is obtained as: $i m f_{1}(x)=\operatorname{res}(x)$; otherwise $\operatorname{res}(x) \leftarrow \operatorname{res}(x)-E_{m n}(x)$ and the above process is repeated until the stopping condition is satisfied.

6. A new signal is generated as $f(x) \leftarrow f(x)-i m f_{1}$, and a new residual is initialised, $r e s(x)=f(x)$, and the process is repeated to derive the next IMF. 
After this sifting process is completed, the original signal $f(x)$ can be decomposed as follows:

$$
f(x)=\sum_{n=1}^{m} i m f_{n}(x)+\operatorname{res}_{m}(x)
$$

where $m$ is the number of IMFs and $r e s_{m}$ is the final residual. The first IMF has the smallest time/space scale. As the decomposition proceeds, the time/space scale increases, and hence, the mean frequency of the mode decreases.

The BEMD is a two-dimensional generalization of the classical EMD, decomposing the original signal into 2-d IMFs, which reduce the frequency information gradually. At each step of the decomposition, the higher-frequency/smaller-scales information is represented by 2-d IMF, while the residual forms the basis of obtaining the following lower-frequency/largerscale 2D-IMF. The original data is recoverable in the same sense as eq. 14.

As for the 1-d signal, the 2-d IMFs depend to the interpolation function used to construct the envelopes, in the present study, surface fitting is based on the thin-plate function ${ }^{31,32}$, and ensure that the gradient is as smooth as possible everywhere.

After the sifting process is completed, the original signal (or image) $f(x, y)$ can be decomposed as follows:

$$
f(x, y)=\sum_{n=1}^{m} 2 D-I M F_{n}(x, y)+r e s_{m}(x, y)
$$

The stopping criterion is again based on the normalized mean-squares difference between two consecutive residuals.

In the present application of the method, the field $f(x, y)$ was decomposed into 3 modes, i.e.

$$
f(x, y)=\sum_{n=1}^{3} 2 D-I M F_{n}(x, y)+r e s_{m}(x, y)
$$

The last residual, $\operatorname{res}_{3}(x, y)$, is treated as the largest-scale mode 4 .

1 K. N. Rao, R. Narasimha, and M. Narayanan, Journal of Fluid Mechanics 48, 339 (1971).

2 P. R. Bandyopadhyay and A. Hussain, Physics of Fluids (1958-1988) 27, 2221 (1984).

3 R. J. Adrian, C. D. Meinhart, and C. D. Tomkins, Journal of Fluid Mechanics 422, 1 (2000).

4 B. Ganapathisubramani, E. K. Longmire, and I. Marusic, Journal of Fluid Mechanics 478, 35 (2003).

5 J. C. Del Álamo and J. Jiménez, Physics of Fluids 15, L41 (2003).

6 J. Jiménez, J. C. Del Alamo, and O. Flores, Journal of Fluid Mechanics 505, 179 (2004).

7 S. Hoyas and J. Jiménez, Physics of fluids 18, 011702 (2006).

8 B. McKeon and J. Morrison, Philosophical Transactions of the Royal Society A: Mathematical, Physical and Engineering Sciences 365, 771 (2007).

9 D. Chung and B. McKeon, Journal of Fluid Mechanics 661, 341 (2010).

10 P. Schlatter, Q. Li, G. Brethouwer, A. V. Johansson, and D. S. Henningson, International Journal of Heat and Fluid Flow 31, 251 (Jun. 2010). 
11 P. H. Alfredsson, A. Segalini, and R. Örlü, Physics of Fluids (1994-present) 23, 041702 (2011).

12 I. Marusic, Physics of fluids 13, 735 (2001).

13 N. Hutchins and I. Marusic, Journal of Fluid Mechanics 579, 1 (2007).

14 R. Mathis, J. P. Monty, N. Hutchins, and I. Marusic, Physics of Fluids 21, 111703 (2009).

15 R. Mathis, N. Hutchins, and I. Marusic, Journal of Fluid Mechanics 628, 311 (2009).

16 I. Marusic, R. Mathis, and N. Hutchins, Science 329, 193 (2010).

17 I. Marusic, R. Mathis, and N. Hutchins, International Journal of Heat and Fluid Flow 31, 418 (2010).

18 I. Marusic, B. J. McKeon, P. A. Monkewitz, H. M. Nagib, A. J. Smits, and K. R. Sreenivasan, Physics of Fluids 22, 065103 (2010).

19 N. Hutchins, J. P. Monty, B. Ganapathisubramani, H. C. H. Ng, and I. Marusic, Journal of Fluid Mechanics 673, 255 (2011).

20 R. Mathis, I. Marusic, S. Chernyshenko, and N. Hutchins, Journal of Fluid Mechanics 715, 163 (2013).

21 P. Schlatter and R. Örlü, Physics of Fluids 22, 051704 (2010).

22 M. Bernardini and S. Pirozzoli, Physics of Fluids 23, 061701 (2011).

23 R. Mathis, N. Hutchins, and I. Marusic, Journal of Fluid Mechanics 681, 537 (2011).

24 S. Chernyshenko, I. Marusic, and R. Mathis, arXiv:1203.3714 [physics](2012).

25 L. Agostini, E. Touber, and M. A. Leschziner, Journal of Fluid Mechanics 743, 606 (2014).

26 E. Touber and M. A. Leschziner, Journal of Fluid Mechanics 693, 150 (2012).

27 P. Luchini (1996).

28 R. D. Moser, J. Kim, and N. N. Mansour, Physics of Fluids 11, 943 (1999).

29 S. Hoyas and J. Jiménez, Physics of Fluids 20, 101511 (Oct. 2008).

30 N. E. Huang, Z. Shen, S. R. Long, M. C. Wu, H. H. Shih, Q. Zheng, N.-C. Yen, C. C. Tung, and H. H. Liu, Proceedings of the Royal Society of London. Series A: Mathematical, Physical and Engineering Sciences 454, 903 (1998).

31 F. Bookstein, IEEE Transactions on Pattern Analysis and Machine Intelligence 11, 567 (Jun. 1989).

32 J. Nunes, Y. Bouaoune, E. Delechelle, O. Niang, and P. Bunel, Image and vision computing 21, 1019 (2003). 